APJ, IN PREP.

Preprint typeset using $\mathrm{LAT}_{\mathrm{E} X}$ style emulateapj v. 8/13/10

\title{
A COMPREHENSIVE ARCHIVAL SEARCH FOR COUNTERPARTS TO ULTRA-COMPACT HIGH VELOCITY CLOUDS: FIVE LOCAL VOLUME DWARF GALAXIES
}

D. J. Sand, ${ }^{1}$ D. Crnojević, ${ }^{1}$ P. Bennet, ${ }^{1}$ B. Willman, ${ }^{2}$ J. Hargis ${ }^{2}$ J. Strader ${ }^{3}$ E. Olszewski, ${ }^{4}$ E.J. Tollerud ${ }^{5,12}$ J.D. Simon, ${ }^{6}$ N. Caldwell, ${ }^{8}$ P. Guhathakurta, ${ }^{10}$ B. L. James, ${ }^{7}$ S. Koposov, ${ }^{7}$ B. Mcleod, N. Morrell ${ }^{9}$, M. Peacock, R. Salinas, ${ }^{3}$ A.C. Seth, ${ }^{11}$ D. P. Stark, ${ }^{4}$ E. Toloba ${ }^{1}$

ApJ, in prep.

\begin{abstract}
We report five Local Volume dwarf galaxies (two of which are presented here for the first time) uncovered during a comprehensive archival search for optical counterparts to ultra-compact high velocity clouds (UCHVCs). The UCHVC population of HI clouds are thought to be candidate gas-rich, low mass halos at the edge of the Local Group and beyond, but no comprehensive search for stellar counterparts to these systems has been presented. Careful visual inspection of all publicly available optical and ultraviolet imaging at the position of the UCHVCs revealed six blue, diffuse counterparts with a morphology consistent with a faint dwarf galaxy beyond the Local Group. Optical spectroscopy of all six candidate dwarf counterparts show that five have an $\mathrm{H} \alpha$-derived velocity consistent with the coincident HI cloud, confirming their association; the sixth diffuse counterpart is likely a background object. The size and luminosity of the UCHVC dwarfs is consistent with other known Local Volume dwarf irregular galaxies. The gas fraction $\left(M_{H I} / M_{\text {star }}\right)$ of the five dwarfs are generally consistent with that of dwarf irregular galaxies in the Local Volume, although ALFALFA-Dw1 (associated with ALFALFA UCHVC HVC274.68+74.70-123) has a very high $M_{H I} / M_{s t a r} \sim 40$. Despite the heterogenous nature of our search, we demonstrate that the current dwarf companions to UCHVCs are at the edge of detectability due to their low surface brightness, and that deeper searches are likely to find more stellar systems. If more sensitive searches do not reveal further stellar counterparts to UCHVCs, then the dearth of such systems around the Local Group may be in conflict with $\Lambda$ CDM simulations. Subject headings: galaxies: dwarf - galaxies: individual (ALFALFA-Dw1, GALFA-Dw1, GALFADw2, GALFA-Dw3, GALFA-Dw4) — radio lines: galaxies
\end{abstract}

\section{INTRODUCTION}

Blind HI surveys have long been used as a foundation for studies of low surface brightness (LSB) galaxies (e.g. Zwaan et al. 1997; Dovle et al. 2005; Rosenberg et al. 2005; West et al. 2010; Cannon et al. 2011; Haynes et al. 2011). Selecting galaxies on the basis of HI mitigates against the optical bias toward galaxies with high surface brightness, and allows targeted follow-up to discover new LSB galaxies (e.g. Spitzak \& Schneider 1998). Extragalactic HI clouds without obvious optical counterparts are themselves interesting probes of (i) our under-

\footnotetext{
david.sand@ttu.edu

${ }^{1}$ Texas Tech University, Physics Department, Box 41051, Lubbock, TX 79409-1051, USA

${ }^{2}$ Department of Physics, Haverford College, 370 Lancaster Avenue, Haverford, PA 19041, USA

3 Michigan State University, Department of Physics and Astronomy, East Lansing, MI 48824, USA

${ }^{4}$ University of Arizona, Tucson, AZ 85721, USA

5 Yale University, Astronomy Department, PO Box 208101, New Haven, CT 06510, USA

${ }^{6}$ Observatories of the Carnegie Institution for Science, 813 Santa Barbara Street, Pasadena, CA 91101, USA

7 Institute of Astronomy, University of Cambridge, Madingley Road, Cambridge, CB3 0HA

8 Harvard-Smithsonian Center for Astrophysics, Cambridge, MA 02138, USA

${ }^{9}$ Las Campanas Observatory, Carnegie Observatories, Casilla 601, La Serena, Chile

10 UCO/Lick Observatory, University of California, Santa Cruz, 1156 High Street, Santa Cruz, CA 95064, USA

11 Department of Physics and Astronomy, University of Utah, Salt Lake City, UT 84112, USA

12 Hubble Fellow
}

standing of the extreme limits of galaxy formation and (ii) our census of dark matter halos in the Universe (e.g. Adams et al. 2015; Cannon et al. 2015; Janowiecki et al. 2015). Such objects may be "dark galaxies": dark matter halos with few or no stars.

Interest in this interpretation has been stoked by tensions between our expectations and observations of the Universe on sub-galactic scales. For example, the discovery of numerous ultra-faint dwarf galaxies around the Milky Way and M31 have underscored the vast incompleteness in our census of dwarf galaxies in even the local universe (e.g. Willman 2010; Hargis et al. 2014). This limits our ability to understand the origin of the "missing satellite problem": the discrepancy between the small number of dwarfs observed around the Milky Way and M31 and the huge number of dark matter sub-halos predicted by theory (e.g. Klypin et al. 1999; Moore et al. 1999).

The advantage of HI galaxy selection is that it offers an opportunity to overcome this incompleteness for nearby LSB galaxies (trading the disadvantage that some LSB galaxies have little or no HI gas). HI clouds are intimately tied to the history of the "missing satellite problem": an early solution was that a subset of the high-velocity HI clouds (HVCs) observed in the Local Group could inhabit some of the "missing" dark matter halos (Braun \& Burton 1999; Blitz et al. 1999). These original studies focused on the HVC catalog of Wakker \& van Woerden (1991) and the HI cloud catalog from the Leiden Dwingeloo Survey (LDS; Hartmann \& Burton 1997), moving later to 
the HI Parkes All Sky Survey catalog (Putman et al. 2002). Many HVCs from these catalogs were studied unsuccessfully for optical emission (Davies et al. 2002; Simon \& Blitz 2002; Willman et al. 2002; Hopp et al. 2003; Siegel et al. 2005; Hopp et al. 2007).

The Galactic Arecibo L-Band Feed Array HI (GALFAHI) and Arecibo Legacy Fast Arecibo L-band Feed Array (ALFALFA) surveys provide a new opportunity for discovering Local Volume galaxies within $\sim 10 \mathrm{Mpc}$. Their spatial resolutions $\left(\sim 4^{\prime}\right.$ for both) and sensitivity $\left(M_{H I} \sim 10^{5}-10^{6} \mathrm{M}_{\odot}\right.$ at $1 \mathrm{Mpc}$ for both) are well-matched to the expected HI properties of star-poor Local Group low-mass halos (Giovanelli et al. 2005; Peek et al. 2011). Catalogs of "ultra-compact" high velocity HI clouds, considered to be the best Local Volume galaxy candidates, have been published for both GALFA-HI's Data Release 1 (58\% of the full survey, Peek et al. 2011) and ALFALFA's $\alpha .40$ HI Source Catalog (Haynes et al. 2011, $40 \%$ of the full survey).

Saul et al. (2012, S12 hereafter) identified a set of 27 UCHVC "galaxy candidates" from their GALFA-HI compact cloud catalog. These objects were selected based on their velocity $\left(\left|V_{L S R}\right|>90 \mathrm{~km} \mathrm{~s}^{-1}\right)$ and lack of association with any known HVC complex or galaxy. Most were barely resolved in the survey data, with a median size of 5'. Adams et al. 2013, A13 hereafter) extracted 59 UCHVCs from ALFALFA's $\alpha .40$ catalog based on their velocity $\left(\left|V_{L S R}\right|>120 \mathrm{~km} \mathrm{~s}^{-1}\right)$ and size; the median size of the ALFALFA UCHVC catalog objects is 12', larger than the GALFA-HI candidates. Nonetheless, there is some overlap between the GALFAHI and ALFALFA UCHVC compilations - eleven ALFALFA UCHVCs are in the GALFA-HI compact cloud catalog, but only one of these is in the "galaxy candidate" group identified by the GALFA-HI team. These differences indicate the difficulty in defining what may be an intrinsically heterogeneous class of objects. Between the two catalogs, there are a total of 85 unique UCHVCs.

The spatial distribution and physical properties of these UCHVCs appear broadly consistent with the hypothesis that some of them are inhabited by optically-faint dwarf galaxies in the Local Volume (Giovanelli et al. 2010; Adams et al. 2013; Faerman et al. 2013). Garrison-Kimmel et al. (2014) also predict that a subset of the A13 UCHVCs are Local Group inhabitants, and that the radial velocities of individual UCHVCs can serve as an initial guess as to which are most likely to host Local Group dwarf galaxies.

That one dwarf galaxy has already been discovered as an optical counterpart to an ALFALFA UCHVC supports the hypothesis that some UCHVCs inhabit Local Volume dark matter halos. The gas-rich Leo $\mathrm{P}$ dwarf galaxy resides at $\mathrm{D} \sim 1.7 \mathrm{Mpc}$, has a $M_{V}=-9.4$ and contains $M_{\mathrm{HI}} \sim 3.6 \times 10^{5} \mathrm{M}_{\odot}$ (Giovanelli et al. 2013; Rhode et al. 2013; McQuinn et al. 2013). Spectroscopic studies have indicated that it is an extremely metaldeficient galaxy (Skillman et al. 2013), with a rotation speed of $15 \mathrm{~km} / \mathrm{s}$, suggesting it lives in a dark matter halo similar to those surrounding faint dwarf galaxies in the Local Group (Bernstein-Cooper et al. 2014). Inspired by this discovery, several groups have recently pursued a combination of archival SDSS and targeted optical obser- vations of S12 and A13's UCHVCs, and discovered dwarf galaxies associated with three of them (Bellazzini et al. 2014; Tollerud et al. 2015, hereafter B14 and T15, respectively). However, no complete UV/optical archival search of S12's and A13's 85 UCHVCs has yet been conducted.

In this paper we present the results of a comprehensive search for optical counterparts to UCHVCs, using both archival and new data. We complement this archival search with new optical imaging and spectroscopic follow-up of all of our viable dwarf galaxy candidates. In Section 2 we discuss our systematic search for dwarf galaxy counterparts. We present optical spectroscopy of our dwarf galaxy counterparts in Section 3 . in order to confirm their association with the UCHVC. In Section 4 we discuss the overall properties of our new dwarf galaxies. We present our conclusions in Section 5 .

When necessary, we adopt a Hubble constant of $H_{0}=$ $69.3 \mathrm{~km} \mathrm{~s}^{-1} \mathrm{Mpc}^{-1}$ from WMAP9 (Hinshaw et al. 2013)

\section{A SEARCH FOR UCHVC DWARF COUNTERPARTS}

In this section we describe our search of publicly available UV/optical data to find UCHVC dwarf galaxy counterparts. In addition to this archival data, we present some complementary deep imaging (Section 2.2) to both search for dwarfs to deeper limits and to confirm dwarf candidates in shallow archival imaging. Our imagingbased search has uncovered six dwarf galaxy candidates, all of which we present spectra for in Section 3. Five are confirmed to have optical velocities consistent with the $\mathrm{HI}$ velocity, and are thus true dwarf galaxy counterparts to the UCHVC.

\subsection{A Visual Search for Dwarf Counterparts in Archival Data}

We have searched all available optical and ultraviolet imaging archives for counterparts to the UCHVCs. In the optical, these include the Digitized Sky Survey (DSS), the Sloan Digital Sky Survey (SDSS Data Release 10; Ahn et al. 2014), the Subaru SMOKA data archive (Baba et al. 2002) and the Canada France Hawaii Telescope's Megacam archive (utilizing the MegaPipe data products; Gwyn 2008). In the ultraviolet, we have made extensive use of the Galaxy Evolution Explorer (GALEX; Martin et al. 2005) archive, which is especially good for uncovering young star forming regions, as is expected for any gas-rich dwarf galaxy the UCHVCs may harbor. We also searched for Swift UltraViolet and Optical Telescope (UVOT) imaging coincident with our UCHVC candidates, but none were found.

Our search is focused on diffuse counterparts to the UCHVC sources, rather than resolved stars. As our guide, we used the visual appearance of Leo $\mathrm{P}$ in the SDSS archive - blue in color (due to recent star formation), blobby and diffuse, with possible O-stars or unresolved HII regions. When multiple optical bands are available, color images were made, facilitating a search for blue counterparts. We confined our visual search for these features to within the $\sim 1$ arcmin positional uncertainty of the UCHVC's position (see A13 and S12), rather than searching the entire extent of the HI cloud, similar to what has been done in past searches for optical counterparts to HI clouds (e.g. Haynes et al. 2011). This corresponds to $\sim 290 \mathrm{pc}$ at $1 \mathrm{Mpc}$. Despite this formal 
$\sim 1$ arcmin search radius, diffuse dwarfs that were $\sim 2-3$ arcmin offset from the HI position would have been recognized during the visual inspection of the images, but none were found. All sources in optical bands with an apparent size $\gtrsim 5$ " were flagged, but most of these extended objects appeared to be background objects and were discarded, with clear spiral/elliptical morphologies, rather than the diffuse nature that we expected. Diffuse galaxies with known velocities that did not agree $(\Delta v>1000$ $\mathrm{km} \mathrm{s}^{-1}$ ) with the UCHVC velocity were also discarded. Any available UV imaging was used to confirm and support dwarf galaxy candidate selection, as the presence of UV flux is expected from a young star forming galaxy. Multiple members of our team searched each image by eye (DJS, DC, PB), with no disagreement between the searchers on dwarf candidates.

Table1 1etails the results of this archival search, which was supplemented by a handful of new images presented in Section 2.2. For data collected from archives other than SDSS and DSS, we have noted the image exposure lengths (or typical survey exposure times) of our searched fields. For instance, in the footnotes to Table 1, we note which UCHVC fields have coverage in the GALEX All-Sky Imaging Survey (AIS) or the Medium Imaging Survey (MIS); see Morrissey et al. (2007) for details on these GALEX surveys. The exposure times for the CFHT imaging are also presented in the footnotes of the table.

Our search uncovered six strong dwarf galaxy candidates, shown in Figures 1 and 2, five of which we were able to confirm with optical spectroscopy were at the same velocity as the UCHVC cloud (see Section 3). For these five dwarfs, we assign names according to the discovering HI survey, in order of increasing right ascension; e.g. GALFA-Dw1, GALFA-Dw2, etc. Two of these, GALFA-Dw1 and GALFA-Dw2, were identified previously by T15 as Pisces A and Pisces B, respectively. A third, ALFALFA-Dw1, was identified and spectroscopically confirmed by Bellazzini et al. (2015) and named SECCO 1 - we confirm its optical velocity is consistent with the UCHVC velocity in Section 3, Given that these dwarfs are not likely to be Local Group members (Section 4.1) and future workers are likely to find large numbers of Local Volume dwarf galaxies (e.g. James et al. 2014), we prefer this nomenclature to, for instance, naming the systems after the corresponding constellation. The current naming scheme also gives credit to the appropriate HI survey.

In the Appendix, we also note several clarifications uncovered during the archival search; including sources that have been previously identified in the literature, or that are associated with known systems. We hope that these clarifications will help future, deeper searches for UCHVC counterparts in prioritizing fields.

\subsection{Supplementary Imaging}

In addition to our archival search for counterparts to the UCHVCs, we have also acquired some supplementary imaging of select systems, either to search for dwarf counterparts to deeper limits or to confirm tentative candidates in shallower archival images. A log of these observations is presented in Table 2, in the order that the data was taken.
HVC274.68+74.70-123 (ALFALFA-Dw1) - We have collected complementary information on this strong dwarf candidate, which we have dubbed ALFALFA-Dw1 (see Figure 11). Images were taken on 11 June 2013 (UT) utilizing Magellan/Megacam (McLeod et al. 2015) with exposures of $8 \times 300 \mathrm{sec}$ in $g$ and $7 \times 300$ in $r$; the conditions were non-photometric. This object was also flagged by B14 as a possible dwarf counterpart to the UCHVC, and was confirmed spectroscopically in Bellazzini et al. (2015). As the CMD of this dwarf has been presented by B14, we do not reproduce it here, but we draw very similar conclusions. Beyond the information reported on this source by Bellazzini et al. (2015), we note a possible second knot of blue optical sources (and UV emission) roughly $\sim 2$ ' Northeast of the main body of this dwarf (see Figure 1). While we do not address this secondary knot further in this work, our upcoming Hubble Space Telescope observations will have full coverage of the ALFALFA-Dw1 field (PID: 13735; PI: Sand). Based on the lack of a tip of the red giant branch (TRGB), we can tentatively place this dwarf beyond $D \sim 3 \mathrm{Mpc}$. We also present our optical velocity measurement in Section 3, and discuss ALFALFA-Dw1's physical properties in later sections.

HVC 351.17+58.56+214/GALFA $215.9+04.6+205$ This UCHVC was flagged by both teams (S12, A13), and was mentioned by A13 in particular for having HI properties similar to Leo $\mathrm{T}$ and Leo $\mathrm{P}$, with both a high column density $\left(N_{H I}>10^{19} \mathrm{~cm}^{-2}\right)$ and a small angular diameter $\left(<7^{\prime}\right)$. Archival observations include SDSS and GALEX AIS NUV, FUV imaging. We imaged HVC351.17+58.56+214 on 27 April 2014 (UT) with Magellan/Megacam. The total exposure time was $6 \times 300$ $\mathrm{s}$ in both the $g$ and $r$ band, taken in photometric conditions, with a limiting magnitude of $r_{0} \sim 25.9 \mathrm{mag}$ (see Crnojević et al. 2014, for data reduction details). No clustering of red or blue stars is apparent in the color magnitude diagram at the position of the HI cloud, and we estimate a lower limit of $D \sim 4 \mathrm{Mpc}$ for any coincident dwarf galaxy.

GALFA 044.7+13.6+528 (GALFA-Dw3) - Inspection of the DSS image at the position of this HI cloud reveals a clear low surface brightness dwarf candidate, which we have dubbed GALFA-Dw3. As such, we imaged the field on 26 October 2014 (UT) with Magellan/Megacam with exposures of $8 \times 300 \mathrm{~s}$ in $r$ and $7 \times 300 \mathrm{~s}$ in $g$. The night was not photometric, and without SDSS coverage, we used the AAVSO Photometric All-Sky Survey (APASS; Henden et al. 2012) to roughly calibrate the field, with a limiting magnitude of $r_{0} \sim 25.5 \mathrm{mag}$. The dwarf is only semi-resolved and crowded in the central regions, and we are unable to accurately determine the stellar population, or measure a TRGB distance. Based on this and the limiting depth of the observations, we conservatively estimate that this dwarf can not be closer than $D \sim 3$ Mpc. The optical velocity of this dwarf is presented in Section 3 .

HVC131.90-46.50-276 - This UCHVC was imaged on 17 November 2014 (UT) with the Seaver Prototype Imaging camera (SPICAM) at the $3.5 \mathrm{~m}$ Astrophysical Research Consortium Telescope at Apache Point Observatory (APO). Images in $g$ and $r$ band were taken with exposure times of $2 \times 540$ s in each band. The data were reduced in a standard way and data from each band were 
combined and astrometrically corrected using SWARF ${ }^{13}$ and astrometry.net (Lang et al. 2010). The data were photometrically calibrated directly to the SDSS system with overlapping stars in the field. Due to poor seeing $\left(\sim 4^{\prime \prime} .5\right)$, the data have a depth of $g \sim 23.3$ and $r \sim 23.5 \mathrm{mag}$, with no sign of a diffuse counterpart to the UCHVC, or of an over density of point sources.

GALFA 086.4+10.8+611 (GALFA-Dw4) - DSS imaging at the position of this UCHVC showed a clear candidate dwarf galaxy. Follow up imaging of this dwarf candidate were taken with APO/SPICAM on 17 November 2014 (UT) in $g$ and $r$ band, with exposures of $4 \times 450$ s in each filter. The data were reduced identically to that of HVC131.90-46.50-276 described above. The data were photometrically calibrated using zero points and color terms derived from two standard star fields taken during the night at different airmasses. We again note that the seeing was poor $(\sim 4$ ") and variable, and so no resolved structure is apparent in this dwarf beyond two 'blobs'. A spectrum and optical velocity is presented in Section 3 .

\section{SPECTROSCOPY}

We have obtained spectroscopic observations for all six of the strong dwarf galaxy candidates identified in our search. Of these six (see Figures 1 and 2), five clearly exhibit $\mathrm{H} \alpha$ with a velocity consistent with that of the UCHVC, confirming them as dwarf counterparts. The sixth object (GALFA $162.1+12.5+434$ ), with a single faint emission line, is likely not associated with the coincident UCHVC. In this section, we detail our spectroscopic observations of all of our dwarf galaxy candidates. An observational log can be see in Table 3, and the spectra of the five confirmed dwarfs are presented in Figure 3 .

ALFALFA-Dw1 (HVC274.68+74.70-123) was observed with the Inamori-Magellan Areal Camera and Spectrograph (IMACS; Dressler et al. 2011) on the Magellan Baade telescope on 17 June 2014 (UT). We obtained five 1800s exposures with the 300 line grating and a 0.77 slit, with wavelength coverage from $\sim 3300-9600$ $\AA$. A resolution of $\sim 2.7 \AA$ was measured from unblended sky lines near the wavelength of $H \alpha$. The slit was oriented along the dwarf, revealing spatially extended $\mathrm{H} \alpha$ emission across $\sim 12$ ", along with $\mathrm{H} \beta$ and the $[\mathrm{O}$ III] $\lambda \lambda 4959,5007$ doublet. An extraction of the full emission yielded an $\mathrm{H} \alpha$ radial velocity (LSR) of $-114 \pm 12$ $\mathrm{km} \mathrm{s}^{-1}$, fully consistent with the HI velocity of $-123 \mathrm{~km}$ $\mathrm{s}^{-1}$. We clearly identify the two $\mathrm{H} \alpha$ knots discussed by Bellazzini et al. (2015), but do not recover the large velocity difference that they see, and which they attribute to mis-centering in the slit.

GALFA-Dw1 (GALFA source 003.7+10.8+236), also known as Pisces A was observed on 29 June 2014 (UT) using the Goodman High-Throughput Spectrograph (Clemens et al. 2004) on the SOAR 4.1-m telescope. We obtained five 900 sec exposures with the 400 $1 \mathrm{~mm}^{-1}$ grating and a $1.03^{\prime \prime}$ slit, yielding a resolution of $5.7 \AA$ and wavelength coverage from $\sim 3100-7000 \AA$. The longslit was oriented at the parallactic angle at the start of the first exposure, and covered both the brightest knot and the main body of the candidate galaxy. Spatially extended emission consistent with the expected position of

13 http://astromatic.iap.fr/software/swarp/
$\mathrm{H} \alpha$ was apparent on the resulting two-dimensional spectra over the location corresponding to the galaxy. No spectral features were associated with the knot itself. Guided by the distribution of the putative $\mathrm{H} \alpha$ emission, we extracted spectra in a $4.8^{\prime \prime}$ aperture centered on the spatial $\mathrm{H} \alpha$ peak emission. The only significant emission line in the spectra is the bright $\mathrm{H} \alpha$ line, from which we derive a radial velocity (LSR) of $236 \pm 8 \mathrm{~km} \mathrm{~s}^{-1}$ through cross-correlation with a rest-frame emission template. This velocity is consistent with both the GALFA $\mathrm{HI}$ velocity (235.37 $\mathrm{km} \mathrm{s}^{-1}$ ) and the optical $\mathrm{H} \alpha$ velocity reported by $\mathrm{T} 15$.

GALFA-Dw2 (GALFA source 019.8+11.1+617), also known as Pisces B, was observed on 21 October 2014 (UT) with the Blue Channel Spectrograph on the MMT (Schmidt et al. 1989). A single $900 \mathrm{sec}$ exposure was obtained with the $300 \mathrm{lmm}^{-1}$ grating and a $1^{\prime \prime}$ slit. The central wavelength was $5300 \AA$, with an order-blocking filter $(<3600 \AA)$, yielding a wavelength range of 3600 $8000 \AA$. A resolution of $\sim 7 \AA$ was measured from unblended sky lines. Spatially extended emission was detected across the face of the dwarf, with emission lines visible in two extended knots. Emission lines corresponding to $\mathrm{H} \alpha, \mathrm{H} \beta$ and the [O III] $\lambda \lambda 4959,5007$ doublet are visible in the spectrum. We extracted a spectrum from one of the knots, and measure a $\mathrm{H} \alpha$ radial velocity of $v_{L S R}=625 \pm 30 \mathrm{~km} \mathrm{~s}^{-1}$. Again, this velocity is consistent with both the GALFA HI velocity $\left(611.63 \mathrm{~km} \mathrm{~s}^{-1}\right)$ and the optical $\mathrm{H} \alpha$ velocity reported by $\mathrm{T} 15$.

GALFA-Dw3 (GALFA source 044.7+13.6+528) and GALFA-Dw4 (GALFA source 086.4+10.8+611) were observed with the $3.5 \mathrm{~m}$ Astrophysical Research Consortium Telescope at Apache Point Observatory (APO) on 18 November 2014 (UT) using the Dual Imaging Spectrograph (DIS). The APO+DIS observations were taken with a 1'.5 slit and the B400/R300 gratings on the red/blue arms of the instrument; wavelength calibration and flat fields were taken at the science position. The position angle for each observation was oriented to maximize the amount of the galaxy (and any knots it may contain) in the slit. The spectrum of GALFA-Dw3 exhibits a clear $\mathrm{H} \alpha$ line, along with faint $\mathrm{H} \beta$ and $[\mathrm{O}$ III] $\lambda \lambda 4959,5007$. With our spectroscopic setup, the $\mathrm{H} \alpha$ is concentrated in a single knot, and does not extend across the body of the dwarf; we measure an $\mathrm{H} \alpha$ velocity of $v_{L S R}=503 \pm 35 \mathrm{~km} \mathrm{~s}^{-1}$, consistent with the UCHVC $\left(v_{L S R}=528.59 \mathrm{~km} \mathrm{~s}^{-1}\right)$. The spectrum of GALFA-Dw4 exhibits $\mathrm{H} \alpha$ emission from two distinct and spatially extended knots; also visible are $\mathrm{H} \beta$, [O III] $\lambda \lambda 4959,5007$, higher order hydrogen Balmer lines, [N II] $\lambda 6584$ and several He I lines. The $\mathrm{H} \alpha$ velocity, $v_{L S R}=607 \pm 35 \mathrm{~km}$ $\mathrm{s}^{-1}$, is consistent with the UCHVC at $v_{L S R}=614.53 \mathrm{~km}$ $\mathrm{s}^{-1}$.

The faint, blue optical source coincident with $G A L F A$ 162.1+12.5+434 (see Figure 2) was observed on 24 December 2014 (UT) with the Blue Channel Spectrograph on the MMT, again with the $300 \mathrm{l} \mathrm{mm}^{-1}$ grating and a $1^{\prime \prime}$ slit, with a total exposure time of $2 \times 900 \mathrm{~s}$. Spatially extended continuum emission was detected across the face of the target, with a single faint emission line. If this emission line is $\mathrm{H} \alpha$, then the velocity of the source is $5700 \pm 200 \mathrm{~km} \mathrm{~s}^{-1}$, where we have taken the mean velocity of the two exposures and their spread as the cen- 
tral value and uncertainty, respectively. If this is the correct line identification, then the blue source seen in Figure 2 is not associated with the HI source GALFA $162.1+12.5+434\left(v_{L S R}=435.67 \mathrm{~km} \mathrm{~s}^{-1}\right)$, and we leave it labeled by the SDSS name of the blue source - SDSS J1048+1230 - in Table 3. We will not discuss it further in this work.

\section{PROPERTIES OF THE DWARFS}

\subsection{Distance Estimates}

The distances to the UCHVC dwarfs are unknown, and will likely require Hubble Space Telescope depth and image quality to determine TRGB distances. Nonetheless, we can estimate upper and lower limits on their distances in order to infer their distance-dependent physical properties in the rest of this section. Our assumed distance range for each dwarf is listed in Table 4.

Both GALFA-Dw1 and GALFA-Dw2 were studied by T15, and we adopt their lower distance bound for our estimates. For GALFA-Dw1, T15 assumed that the lower bound on the distance was $\mathrm{D}=1.7 \mathrm{Mpc}$, based on a comparison of point sources in GALFA-Dw1 with respect to Leo P's main sequence stars. For GALFA-Dw2, T15 assumed a lower distance limit of $\mathrm{D}=3.5 \mathrm{Mpc}$ based on their deep ground-based imaging. As an upper limit for the distance to these dwarfs we adopt a distance which is a factor of 1.5 above the Hubble flow value for each dwarf, in order to be conservative $-\mathrm{D}=5.3 \mathrm{Mpc}$ for GALFADw1 and D=13.4 Mpc for GALFA-Dw2.

Similarly, ALFALFA-Dw1 was recently studied by Bellazzini et al. (2015), and we broadly adopt their distance estimate range. Based off of their Large Binocular Telescope color magnitude diagram (and the lack of a TRGB), they estimate a lower distance limit of $\mathrm{D}=3$ Mpc. Our own Magellan/Megacam color magnitude diagram agrees with this estimate. However, ALFALFADw1 is projected onto the Virgo cluster, and it is possibly a faint, star forming member; this would imply a distance of up to $\mathrm{D}=18 \mathrm{Mpc}$ (using a distance to the Virgo Cluster of $\mathrm{D}=16.5 \mathrm{Mpc}$, with an intrinsic spread in line of sight distances of $\sim 1.5 \mathrm{Mpc}$; Mei et al. 2007).

For both GALFA-Dw3 and GALFA-Dw4, we take a simple approach, assuming that their distance lies somewhere between $\mathrm{D}=3 \mathrm{Mpc}$ (based off of the Magellan/Megacam image depth for GALFA-Dw3) and a factor of 1.5 beyond their Hubble flow distance $-\mathrm{D}=11.4$ Mpc for GALFA-Dw3 and $\mathrm{D}=13.3 \mathrm{Mpc}$ for GALFADw4.

\subsection{Luminosity and Half-Light Radius}

We estimate the magnitude and luminosity of our new dwarfs directly from elliptical aperture photometry, similar to that presented in Sand et al. (2014). We choose an elliptical aperture for each dwarf based on all available imaging (in different bands) such that no dwarf flux extends beyond the aperture. Note that the position angle and ellipticity of the aperture is not constrained directly, but is simply chosen to be in the visible direction of the light profile. Care is taken to avoid bright nearby stars within the aperture, although some contamination is inevitable. The aperture position is adjusted by centroiding on the $r$-band image within an initial elliptical aperture, placed at the approximate center of the dwarf.
We use this optical centroid position as the position of the dwarf, which we report in Table 4, along with the other parameters derived in this Section.

For our optical data, we randomly place $\sim 30-50$ apertures of equal area to that used to measure the dwarf flux throughout the field, in order to estimate the background. This accounts for interloping stars/galaxies in the dwarf aperture in a statistical sense. We report our measured $r_{0}$ and $(g-r)_{0}$ color for each dwarf in Table 4, after accounting for Galactic extinction (Schlafly \& Finkbeiner 2011). For our GALEX UV data, we use the same elliptical aperture to measure the dwarf flux as in the optical, but instead use an elliptical annulus $\sim 20-30 "$ beyond the radius of the dwarf to measure the background. We chose this methodology for the UV data because of the strongly varying image quality across the GALEX focal plane.

The color of the dwarf galaxies are all $(g-r)_{0} \lesssim 0.3 \mathrm{mag}$. Comparison with a set of single stellar population colors as a function of age (Bressan et al. 2012) suggests this corresponds to stellar ages of $\lesssim 1$ Gyr. For those dwarfs with a color of $(g-r)_{0} \sim 0 \mathrm{mag}$ (e.g. ALFALFA-Dw1, GALFA-Dw4, and even the others within the uncertainties) the single stellar population age is nearer $\sim 100 \mathrm{Myr}$. Higher precision photometry is necessary to further constrain the stellar population of these dwarfs.

The half light radius of each dwarf was measured from the $r$-band data. The photometric aperture of each dwarf (and the background apertures) was closed down until half of the total dwarf flux was enclosed, taking into account background uncertainties and the uncertainty in the total magnitude of each dwarf.

We can compare our results for GALFA-Dw1 and GALFA-Dw2 directly with T15, keeping in mind that the current work utilizes SDSS imaging, while T15 used deeper ground-based data. Both sets of measurements are consistent for GALFA-Dw1, to within the 1- $\sigma$ uncertainties, while the measurements for GALFA-Dw2 are consistent within 2- $\sigma$. Given the diffuse nature of these dwarfs, and the different quality datasets, we consider this level of agreement satisfactory. Similarly, comparing our measurements with those of ALFALFA-Dw1 presented in B15, we find agreement within the 1- $\sigma$ uncertainties.

Combining these measurements with our distance estimates presented in Section 4.1. we can constrain the absolute magnitude and physical half light radii of the new dwarfs (see Table 4), and place them into context with respect to other Local Volume dwarfs, as we do in Figure 4. Absolute $r$-band magnitudes were converted into $V$ band using the SDSS filter transformations of Jester et al. (2005). Despite their large distance uncertainties (indicated by the colored bands), the new UCHVC dwarf galaxies are similar to other Local Volume dwarfs with ongoing star formation (e.g. dwarf irregulars) in $M_{V}$ versus $r_{h}$ space. Note that the half light radius for Leo $\mathrm{P}$ has not been measured yet, and so we take the semi-major axis size from McQuinn et al. (2013), which would be an overestimate of the true half light radius.

While a detailed understanding of our dwarf detection limits are difficult to assess given the heterogenous nature of our search, we can make some conservative statements. First, the Local Group dwarf spheroidals, and 
the Local Volume dwarf irregulars, clearly suffer from surface brightness selection effects themselves; we have drawn lines of constant central surface brightness (assuming an exponential profile) in Figure 4, and both groups of dwarfs are likely missing "large and faint" members. Although our archival search was heterogenous, most of our fields were within the SDSS footprint. Given this and our search methodology, where the size of any dwarf counterpart had to be $>5$ ", implies a central surface brightness limit of $\mu_{0} \sim 23.5-24 \mathrm{mag} \operatorname{arcsec}^{-2}$ for a dwarf with a magnitude of $V \sim 17 \mathrm{mag}$ (see Figure (4). In this sense, it is unsurprising that the majority of the dwarfs uncovered have the properties that they do. The case of ALFALFA-Dw1 shows that deeper searches may be fruitful, despite the recent deep imaging of B14 and T15 on a subset of the UCHVC sample; there is no trace of this dwarf in the SDSS imaging.

\subsection{Environment}

Even though their distances are unknown, it may still be possible to glean some clues as to the environment that our new UCHVC dwarfs are in. We have searched the NASA Extragalactic Database for all objects on the sky within 3 degrees $(\sim 500 \mathrm{kpc}$ at $10 \mathrm{Mpc}$ or $\sim 170 \mathrm{kpc}$ at $3 \mathrm{Mpc}$ ) and $400 \mathrm{~km} / \mathrm{s}$ of each of our dwarf discoveries.

A search in the vicinity of ALFALFA-Dw1 (with $v_{L S R}=-123 \mathrm{~km} \mathrm{~s}^{-1}$ ) turns up many counterparts, as it is projected onto the so-called 'Low Velocity Cloud' portion of the Virgo Cluster (at $\mathrm{D}=17 \mathrm{Mpc}$; Gavazzi et al. 1999), which has a velocity distribution centered on $v_{L S R} \sim 0 \mathrm{~km} \mathrm{~s}^{-1}$ with a dispersion of $\sim 200 \mathrm{~km} \mathrm{~s}^{-1}$ (e.g. Boselli et al. 2014). On the other hand, if the secondary blue clump of stars seen in Figure 1 are really associated with ALFALFA-Dw1, then it is possible that this object is nearby, but with a low overall surface brightness. Recent observations of Virgo Cluster galaxy IC 3418 have highlighted an extended tail of UV emission and HII regions spanning similar size scales as ALFALFA-Dw1 and its secondary blue clump, likely due to ram pressure stripping (Kennev et al. 2014), and it is plausible that we are witnessing something similar in ALFALFA-Dw1 and its secondary clump. Upcoming HST Cycle 22 observations will possibly pin down its distance and shed light on the nature of ALFALFA-Dw1.

GALFA-Dw2 is possibly associated with the dwarf galaxy UGC $891\left(M_{V}=-15\right)$, which is $\sim 80$ ' away from it on the sky and has an almost identical velocity $\left(v_{\text {helio }}=643 \mathrm{~km} \mathrm{~s}^{-1}\right)$. The current best distance estimate for UGC 891 is based on the Tully-Fisher relation, at $\mathrm{D}=9.4 \mathrm{Mpc}$ (Tully 1988). Assuming that GALFA-Dw2 and UGC 891 are both at this distance, then they are physically separated by $\sim 230 \mathrm{kpc}$. In this case, it is possible that GALFA-Dw2 belongs to a UGC 891 'dwarf association'; Leo P and several of the HI-rich SHIELD galaxies are also in a similar environment (McQuinn et al. 2013, 2014).

The remaining three UCHVC dwarfs - GALFA-Dw1, GALFA-Dw3 and GALFA-Dw4 - appear to be remarkably isolated, with only a handful of other HI sources (with no known stellar counterpart) turning up in our search. Similarly isolated objects are rare, but are occasionally reported in the literature (e.g. Pasquali et al. 2005; Karachentsev et al. 2014, 2015), and present an opportunity to learn about star formation without any external environmental factors. Again, several of the SHIELD galaxies also appear totally isolated (McQuinn et al. 2014), suggesting that the new UCHVC dwarfs come from broadly similar environments.

\subsection{Gas and Stellar Masses}

HI gas masses, and the gas to stellar mass ratio in particular, tell us about the efficiency of gas conversion to stars in a given galaxy. We compute HI gas masses (using our assumed distance ranges; Section 4.1) directly from the data cubes of the GALFA-HI survey for two reasons. First, a tailored extraction aperture is necessary to capture the entire HI flux from each cloud. Second, the tabulated values in S12 are in error, and will be corrected in an upcoming GALFA HI update (Saul et al., in prep). The GALFA-Dw1 and GALFA-Dw2 HI fluxes are unchanged from T15. As the HI data cubes from A13 are not publicly available, we take the integrated HI flux for ALFALFA-Dw1 directly from that work (integrated HI fluxes using other instrumentation provide consistent results to within $\sim 15 \%$; E. Adams, private communication).

To calculate the stellar mass for our UCHVC dwarf sample, we have assumed that $\left(M_{\text {star }} / L_{V}\right) /\left(M_{\odot} / L_{\odot}\right)=1$ and translated our absolute $\mathrm{V}$-band magnitudes (with better color measurements of these dwarfs, an improved $\left(M_{\text {star }} / L_{V}\right) /\left(M_{\odot} / L_{\odot}\right)$ could be assigned, e.g. Bell et al. 2003). The resulting $M_{H I} / M_{\text {star }}$ versus $M_{\text {star }}$ plot is shown in the right panel of Figure 4, along with similar data for Leo T (Ryan-Weber et al. 2008; de Jong et al. 2008), Leo P, Local Volume dwarf irregulars (with data from McConnachie 2012), the SHIELD sample of HIselected low-mass dwarf galaxies (Cannon et al. 2011; McQuinn et al. 2015), and the pilot sample of 'Almost Dark' galaxies (Cannon et al. 2015). Several of the new dwarfs have similar $M_{H I} / M_{\text {star }}$ values as other Local Volume dwarf irregulars, again suggesting that these may just be standard members of this class that have not been uncovered yet. ALFALFA-Dw1, on the other hand, displays a very large $M_{H I} / M_{\text {star }} \sim 38$, comparable to the 'Almost Dark' galaxies (Cannon et al. 2015; Janowiecki et al. 2015). This emerging class of galaxies may represent the transition between nearly starfree halos with only HI gas (e.g. Adams et al. 2015) and those with a $M_{H I} / M_{\text {star }} \sim 1$, although these systems still have $>100$ times the stellar mass of objects like Segue 1 (Martin et al. 2008) around the Milky Way (MW). Nevertheless, they deserve intensive follow up in the ultraviolet, optical and radio regimes.

\section{CONCLUSIONS}

We have presented a systematic, archival UV/optical search for stellar counterparts to the recently uncovered HI UCHVC population, as these systems may shed light on how stars populate dark matter halos in isolated environments. By searching all available optical and ultraviolet public imaging archives - along with some supplementary imaging - we found six compelling dwarf galaxy candidates coincident with HI UCHVC positions. Spectroscopic followup of all six candidates revealed five with velocities consistent with the HI cloud. We measured the magnitude and half light radius of all five dwarfs, 
and their physical properties appear broadly consistent with the faint end of gas rich stellar systems.

There are many outstanding questions centered on these UCHVC dwarf galaxies. Leading among them are their unknown distances, which will require Hubble Space Telescope imaging, as well as their detailed star formation histories. Another promising path forward is to understand the HII region metallicities and physical conditions of these dwarfs, which will require deep optical spectroscopy; for instance, recent work on Leo $\mathrm{P}$ has shown it to be among the most metal deficient star forming galaxies ever observed (Skillman et al. 2013).

The current sample of UCHVC dwarfs is in some tension with expectations from cosmological simulations. All of them, with the possible exception of GALFADw1, are likely too distant for comparisons with recent simulations meant to resemble the Local Group, such as ELVIS (Garrison-Kimmel et al. 2014). What may be more remarkable is the fact that no dwarf counterparts (again, with the possible exception of GALFA-Dw1) were found that could be plausibly associated with the Local Group. While our search was insensitive to Leo Tlike dwarfs (which is at $\mathrm{D} \sim 400 \mathrm{kpc}$ and is resolved into stars by SDSS), we were sensitive to Leo P-like objects (which is at $\mathrm{D} \sim 1.5 \mathrm{Mpc}$ and appears as a blue diffuse object in SDSS). The predicted HI mass function from ELVIS suggests that $\sim 50$ unidentified dwarf galaxies with $M_{H I}>10^{5} M_{\odot}$ may be within $\mathrm{D} \sim 1.2 \mathrm{Mpc}$ of the MW or M31 (Garrison-Kimmel et al. 2014), which is comparable to the $M_{H I}$ detection limits at that distance for both GALFA-HI and ALFALFA. Given the sky coverage of the GALFA-HI Data Release 1 (18\% of the sky; Peek et al. 2011) and ALFALFA $\alpha .40$ Data Release ( $\sim 7 \%$ of the sky; Haynes et al. 2011), 5-10 dwarf counterparts would thus be expected. Either these potential galaxies a) never formed stars and so would be invisible to our search; b) are too near to us and so would appear as resolved stars rather than diffuse galaxies in our search (and so would have gone undiscovered) or c) there is a conflict with expectations from recent $\Lambda$ CDM simulations. Clearly, distance measurements to the newly discovered dwarfs, and deeper searches for stellar counterparts to the other UCHVCs, are necessary before decisive conclusions can be drawn.

Finally, our archival search for UV/optical counterparts uncovered four dwarf galaxies associated with the GALFA-HI Compact Cloud Catalog (consisting of 27 sources overall), while the ALFALFA UCHVC catalog (consisting of 59 total sources) uncovered only a single object. Some effort was made by the ALFALFA team to not include UCHVCs which had clear optical counterparts in DSS/SDSS into the final UCHVC catalog of A13, so this may be the root of the difference between the two surveys (E. Adams, private communication). Once the GALFA-HI fluxes have been amended, it will be important to do a cross-comparison between the ALFALFA and GALFA UCHVC catalogs, and identify which physi- cal HI-related properties are most important for yielding a dwarf galaxy companion. A broader search of compact HI clouds may be warranted in order to uncover faint dwarf galaxies that may be at the edge of the selection criteria that are currently being employed by the different HI teams. Another path forward is to search the SDSS archives for blue diffuse galaxies (e.g. James et al. 2014), or to examine the GALEX archive. In any case, as ALFALFA-Dw1 has shown, there may yet be several interesting stellar systems associated with the UCHVC population, but below the detection limit of surveys such as SDSS and DSS; further deep followup work is needed.

We warmly thank Maureen Conroy, John Roll and Sean Moran for their prolonged efforts and help related to Magellan/Megacam. We thank Alison Marqusee, Chris Nagele, and Eric Smith for assistance obtaining ARC $3.5 \mathrm{~m}$ observations. We are also grateful to Elizabeth Adams for useful comments. DJS, JDS and PG acknowledge support from NSF grant AST-1412504. BW and JH acknowledge support from NSF AST-1151462. PG acknowledges additional support from NSF grant AST-1010039. EO thanks the NSF for support through AST-1313006. The Digitized Sky Surveys were produced at the Space Telescope Science Institute under U.S. Government grant NAG W-2166. The images of these surveys are based on photographic data obtained using the Oschin Schmidt Telescope on Palomar Mountain and the UK Schmidt Telescope. The plates were processed into the present compressed digital form with the permission of these institutions. This research has made use of the NASA/IPAC Extragalactic Database (NED) which is operated by the Jet Propulsion Laboratory, California Institute of Technology, under contract with the National Aeronautics and Space Administration. Funding for SDSS-III has been provided by the Alfred P. Sloan Foundation, the Participating Institutions, the National Science Foundation, and the U.S. Department of Energy Office of Science. The SDSSIII web site is http://www.sdss3.org/. Based in part on data collected at the Subaru Telescope and obtained from SMOKA, which is operated by the Astronomy Data Center, National Astronomical Observatory of Japan. This research was made possible through the use of the AAVSO Photometric All-Sky Survey (APASS), funded by the Robert Martin Ayers Sciences Fund. Observations reported here were obtained at the MMT Observatory, a joint facility of the University of Arizona and the Smithsonian Institution. This paper includes data gathered with the 6.5 meter Magellan Telescopes located at Las Campanas Observatory, Chile. Based on observations obtained at the Southern Astrophysical Research (SOAR) telescope, which is a joint project of the Ministério da Ciência, Tecnologia, e Inovação (MCTI) da República Federativa do Brasil, the U.S. National Optical Astronomy Observatory (NOAO), the University of North Carolina at Chapel Hill (UNC), and Michigan State University (MSU). 


\section{APPENDIX}

\section{UCHVC CLARIFICATIONS}

A handful of the UCHVC sources have already appeared in the literature or are coincident with known galaxies. We briefly describe each case here.

GALFA $147.0+0 \% .1+525$ - This GALFA nearby galaxy candidate is also in the $\alpha .40$ catalog, listed as AGC 191803 (Havnes et al. 2011). Coincident with the HI source is a blue, disky galaxy, easily detectable in SDSS bands and in GALEX archival data. It also is listed in the Updated Nearby Galaxy Catalog (Karachentsev et al. 2013), which reports a Tully-Fisher distance of $14.9 \mathrm{Mpc}$, whereas the Hubble Flow distance is 7.6 Mpc. While it is likely a formality, to our knowledge there is no $\mathrm{H} \alpha$ velocity confirming the association between the HI source and the blue, coincident galaxy. As this galaxy has been reported elsewhere, we do not include it in our main sample of new discoveries, although further followup is warranted.

GALFA 188.9+14.5+387 - This GALFA nearby galaxy candidate appears coincident with M91, albeit at a somewhat lower velocity $\left(v_{L S R}=403.41 \mathrm{~km} \mathrm{~s}^{-1}\right.$ with FWHM $=68.76 \mathrm{~km} \mathrm{~s}^{-1}$; versus $v_{L S R}=491 \mathrm{~km} \mathrm{~s}{ }^{-1}$ for M91). As pointed out by Grcevich (2013), it is plausible that this gas cloud is an extension of M91's halo or that it is a projected dwarf galaxy.

GALFA $187.5+08.0+473$ - This gas cloud is projected between VCC 1249 and M49, and has been previously identified by McNamara et al. (1994). It is almost certainly stripped gas from a past encounter between VCC 1249 and M49, as has been discuss at length by Arrigoni Battaia et al. (2012).

ALFALFA 320.95+72.32+185 - This gas cloud is projected 1.4' away from UGC 08298, an irregular galaxy with $\mathrm{v}_{\odot}=1157 \mathrm{~km} \mathrm{~s}^{-1}$. It is unlikely that the ALFALFA source, at $v_{L S R}=185 \mathrm{~km} \mathrm{~s}^{-1}$, is associated with UGC08298, given the large velocity difference between the two systems.

\section{REFERENCES}

Adams, E. A. K., Giovanelli, R., \& Haynes, M. P. 2013, ApJ, 768, 77

Adams, E. A. K., Faerman, Y., Janesh, W. F., et al. 2015, A\&A, 573, L3

Ahn, C. P., Alexandroff, R., Allende Prieto, C., et al. 2014, ApJS, 211, 17

Arrigoni Battaia, F., Gavazzi, G., Fumagalli, M., et al. 2012, A\&A, 543, A112

Baba, H., Yasuda, N., Ichikawa, S.-I., et al. 2002, in Astronomical Society of the Pacific Conference Series, Vol. 281, Astronomical Data Analysis Software and Systems XI, ed. D. A. Bohlender, D. Durand, \& T. H. Handley, 298

Bell, E. F., McIntosh, D. H., Katz, N., \& Weinberg, M. D. 2003, ApJS, 149, 289

Bellazzini, M., Beccari, G., Battaglia, G., et al. 2014, ArXiv e-prints, arXiv:1412.5857

Bellazzini, M., Magrini, L., Mucciarelli, A., et al. 2015, ArXiv e-prints, arXiv:1501.06305

Bernstein-Cooper, E. Z., Cannon, J. M., Elson, E. C., et al. 2014, AJ, 148,35

Blitz, L., Spergel, D. N., Teuben, P. J., Hartmann, D., \& Burton, W. B. 1999, ApJ, 514, 818

Boselli, A., Voyer, E., Boissier, S., et al. 2014, A\&A, 570, A69

Braun, R., \& Burton, W. B. 1999, A\&A, 341, 437

Bressan, A., Marigo, P., Girardi, L., et al. 2012, MNRAS, 427, 127

Cannon, J. M., Giovanelli, R., Haynes, M. P., et al. 2011, ApJ, 739, L22

Cannon, J. M., Martinkus, C. P., Leisman, L., et al. 2015, AJ, 149,72

Clemens, J. C., Crain, J. A., \& Anderson, R. 2004, in Society of Photo-Optical Instrumentation Engineers (SPIE) Conference Series, Vol. 5492, Ground-based Instrumentation for Astronomy, ed. A. F. M. Moorwood \& M. Iye, 331-340

Crnojević, D., Sand, D. J., Caldwell, N., et al. 2014, ApJ, 795, L35

Davies, J., Sabatini, S., Davies, L., et al. 2002, MNRAS, 336, 155

de Jong, J. T. A., Harris, J., Coleman, M. G., et al. 2008, ApJ, 680, 1112

Doyle, M. T., Drinkwater, M. J., Rohde, D. J., et al. 2005, MNRAS, 361, 34

Dressler, A., Bigelow, B., Hare, T., et al. 2011, PASP, 123, 288

Faerman, Y., Sternberg, A., \& McKee, C. F. 2013, ApJ, 777, 119

Garrison-Kimmel, S., Boylan-Kolchin, M., Bullock, J. S., \& Lee, K. 2014, MNRAS, 438, 2578

Gavazzi, G., Boselli, A., Scodeggio, M., Pierini, D., \& Belsole, E. 1999, MNRAS, 304, 595
Giovanelli, R., Haynes, M. P., Kent, B. R., \& Adams, E. A. K. 2010, ApJ, 708, L22

Giovanelli, R., Haynes, M. P., Kent, B. R., et al. 2005, AJ, 130, 2598

Giovanelli, R., Haynes, M. P., Adams, E. A. K., et al. 2013, AJ, 146,15

Grcevich, J. 2013, PhD thesis, Columbia University

Gwyn, S. D. J. 2008, PASP, 120, 212

Hargis, J. R., Willman, B., \& Peter, A. H. G. 2014, ApJ, 795, L13

Hartmann, D., \& Burton, W. B. 1997, Atlas of Galactic Neutral Hydrogen

Haynes, M. P., Giovanelli, R., Martin, A. M., et al. 2011, AJ, 142, 170

Henden, A. A., Levine, S. E., Terrell, D., Smith, T. C., \& Welch, D. 2012, Journal of the American Association of Variable Star Observers (JAAVSO), 40, 430

Hinshaw, G., Larson, D., Komatsu, E., et al. 2013, ApJS, 208, 19

Hopp, U., Schulte-Ladbeck, R. E., \& Kerp, J. 2003, MNRAS, 339, 33

-. 2007, MNRAS, 374, 1164

James, B. L., Koposov, S., Stark, D. P., et al. 2014, ArXiv e-prints, arXiv:1411.7371

Janowiecki, S., Leisman, L., Jozsa, G., et al. 2015, ArXiv e-prints, arXiv: 1502.01296

Jester, S., Schneider, D. P., Richards, G. T., et al. 2005, AJ, 130, 873

Karachentsev, I. D., Makarov, D. I., \& Kaisina, E. I. 2013, AJ, 145,101

Karachentsev, I. D., Makarova, L. N., Makarov, D. I., Tully, R. B., \& Rizzi, L. 2014, ArXiv e-prints, arXiv:1411.1674 -. 2015, MNRAS, 447, L85

Kenney, J. D. P., Geha, M., Jáchym, P., et al. 2014, ApJ, 780, 119

Klypin, A., Kravtsov, A. V., Valenzuela, O., \& Prada, F. 1999, ApJ, 522, 82

Lang, D., Hogg, D. W., Mierle, K., Blanton, M., \& Roweis, S. 2010, AJ, 139, 1782

Martin, D. C., Fanson, J., Schiminovich, D., et al. 2005, ApJ, 619, L1

Martin, N. F., de Jong, J. T. A., \& Rix, H.-W. 2008, ApJ, 684, 1075

McConnachie, A. W. 2012, AJ, 144, 4

McLeod, B., Geary, J., Conroy, M., et al. 2015, PASP, 127, 366

McNamara, B. R., Sancisi, R., Henning, P. A., \& Junor, W. 1994, AJ, 108,844

McQuinn, K. B. W., Skillman, E. D., Berg, D., et al. 2013, AJ, 146, 145 
McQuinn, K. B. W., Cannon, J. M., Dolphin, A. E., et al. 2014, ApJ, 785, 3

-. 2015, ArXiv e-prints, arXiv:1501.07313

Mei, S., Blakeslee, J. P., Côté, P., et al. 2007, ApJ, 655, 144

Moore, B., Ghigna, S., Governato, F., et al. 1999, ApJ, 524, L19

Morrissey, P., Conrow, T., Barlow, T. A., et al. 2007, ApJS, 173, 682

Pasquali, A., Larsen, S., Ferreras, I., et al. 2005, AJ, 129, 148

Peek, J. E. G., Heiles, C., Douglas, K. A., et al. 2011, ApJS, 194, 20

Putman, M. E., de Heij, V., Staveley-Smith, L., et al. 2002, AJ, 123,873

Rhode, K. L., Salzer, J. J., Haurberg, N. C., et al. 2013, AJ, 145, 149

Rosenberg, J. L., Schneider, S. E., \& Posson-Brown, J. 2005, AJ, 129, 1311

Ryan-Weber, E. V., Begum, A., Oosterloo, T., et al. 2008, MNRAS, 384, 535

Sand, D. J., Strader, J., Willman, B., et al. 2012, ApJ, 756, 79

Sand, D. J., Crnojević, D., Strader, J., et al. 2014, ApJ, 793, L7
Saul, D. R., Peek, J. E. G., Grcevich, J., et al. 2012, ApJ, 758, 44 Schlafly, E. F., \& Finkbeiner, D. P. 2011, ApJ, 737, 103

Schmidt, G. D., Weymann, R. J., \& Foltz, C. B. 1989, PASP, 101,713

Siegel, M. H., Majewski, S. R., Gallart, C., et al. 2005, ApJ, 623, 181

Simon, J. D., \& Blitz, L. 2002, ApJ, 574, 726

Skillman, E. D., Salzer, J. J., Berg, D. A., et al. 2013, AJ, 146, 3

Spitzak, J. G., \& Schneider, S. E. 1998, ApJS, 119, 159

Tollerud, E. J., Geha, M. C., Grcevich, J., Putman, M. E., \& Stern, D. 2015, ApJ, 798, L21

Tully, R. B. 1988, Nearby galaxies catalog

Wakker, B. P., \& van Woerden, H. 1991, A\&A, 250, 509

West, A. A., Garcia-Appadoo, D. A., Dalcanton, J. J., et al. 2010, AJ, 139, 315

Willman, B. 2010, Advances in Astronomy, 2010, arXiv:0907.4758 Willman, B., Dalcanton, J., Ivezić, Ž., Schneider, D. P., \& York, D. G. 2002, AJ, 124, 2600

Zwaan, M. A., Briggs, F. H., Sprayberry, D., \& Sorar, E. 1997, ApJ, 490, 173 

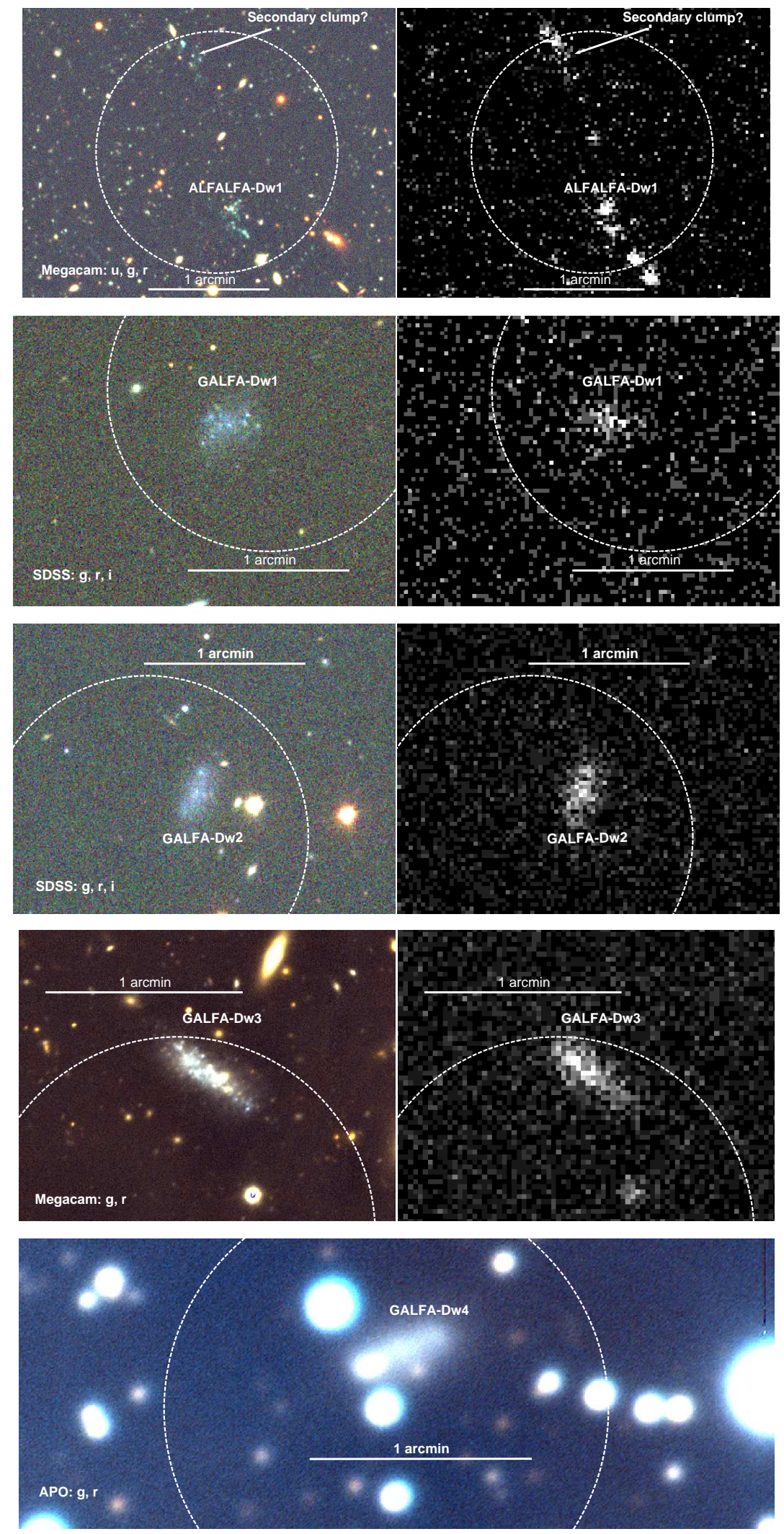

FIG. 1.- Color optical images, as well as GALEX NUV data (right panel, when available) of the five dwarf galaxies uncovered in archival data with optical velocities consistent with their coincident UCHVC cloud. GALFA-Dw4 is 10 degrees from the plane of the Milky Way, and is behind $\sim 1.5-2$ magnitudes of extinction; there is no GALEX UV data. The three-color image for GALFA-Dw4 was constructed using the two available bands, and averaging their flux to create the green channel of the image. All of the dwarf galaxies appear blue in the optical, and have strong GALEX detections, indicating a young stellar population. The dashed circular region roughly represents the HI positional uncertainty. North is up and East is left. 


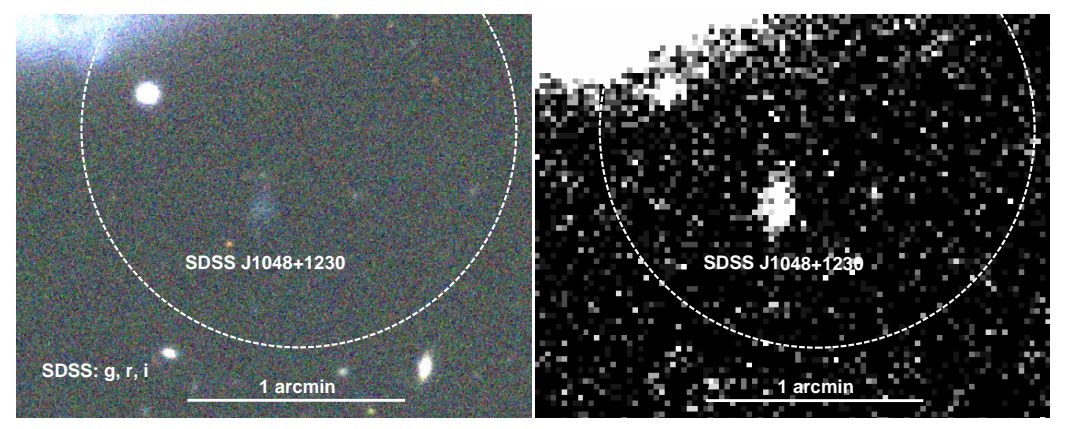

FIG. 2.- An SDSS 3-color image of the dwarf galaxy candidate associated with GALFA 162.1+12.5+434, along with the corresponding GALEX NUV data. The dashed circular region roughly represents the HI positional uncertainty. Follow up spectroscopy showed a single, weak emission line. If this line is associated with $\mathrm{H} \alpha$, the galaxy would have a velocity of $\sim 5700 \mathrm{~km} \mathrm{~s}^{-1}$, and is likely not related to GALFA $162.1+12.5+434$. It is possible that the UCHVC GALFA $162.1+12.5+434$ is associated with the nearby galaxy NGC 3389 (just visible in the Northeast portion of the image), with a velocity difference of $\sim 800 \mathrm{~km} \mathrm{~s}^{-1}$. 

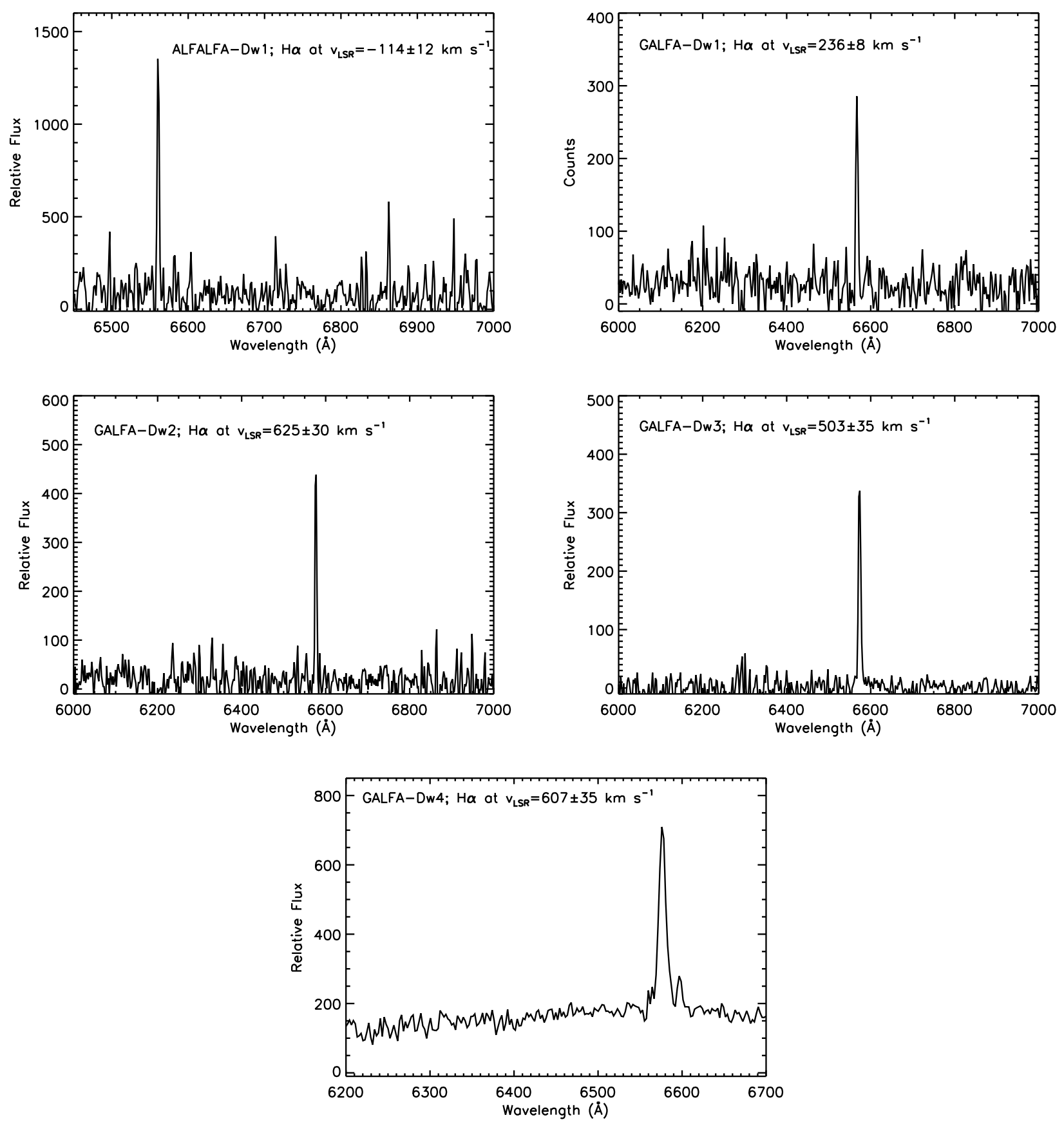

FIG. 3.- Spectroscopic cutouts of the five UCHVC counterparts with $\mathrm{H} \alpha$ velocities consistent with that of HI. Further details for each observation, and other detected optical lines, can be seen in Table 3 and Section 3 

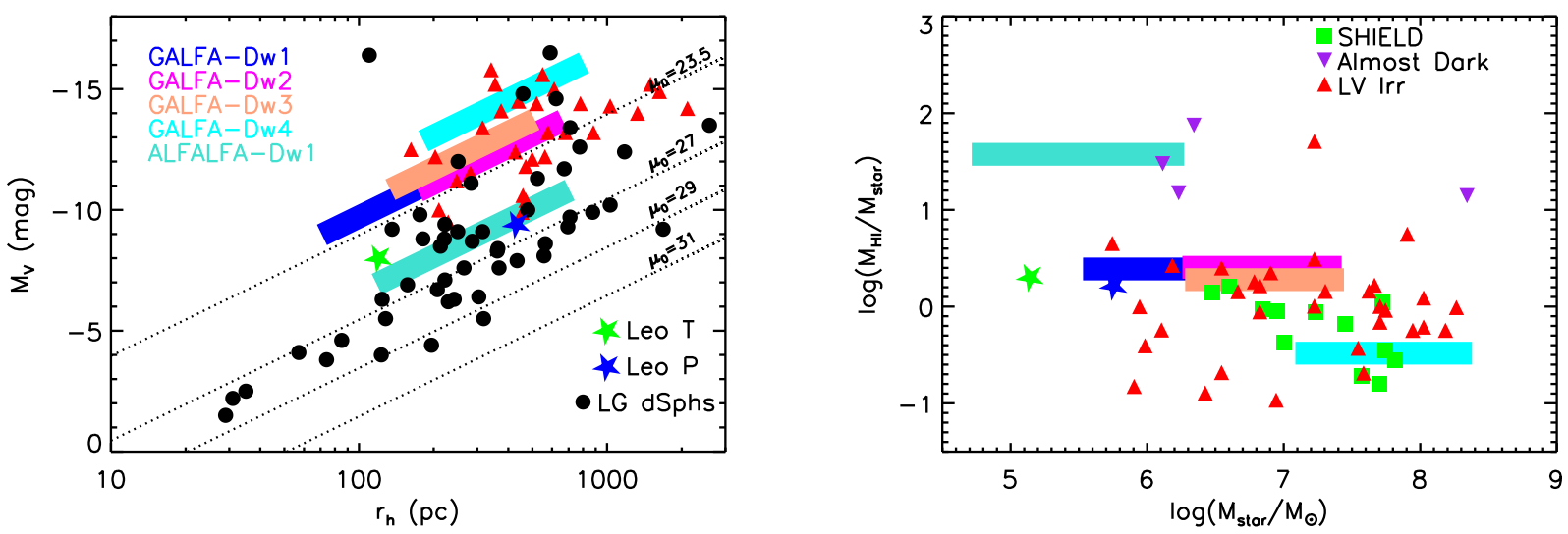

FIG. 4. - Left - Absolute magnitude as a function of half light radius for both Local Group dwarf spheroidals (around the Milky Way and M31) and dwarf irregular galaxies in the Local Volume. All data were taken from McConnachie (2012) and Sand et al. (2012), except for that of Leo P, which is from McQuinn et al. (2013). We draw lines of constant central surface brightness (assuming an exponential profile) in order to emphasize that there are still likely to be selection effects associated with all of the known dwarf galaxy populations, including the current search - see discussion in Section 4.2 The UCHVC dwarfs uncovered by our search, marked by colored bands corresponding to their distance uncertainties, have similar physical properties to those in the Local Volume especially considering the rough surface brightness limit of our search and their current distance uncertainties. Right - The ratio $M_{H I} / M_{\text {star }}$ as a function of stellar mass for various sample of gas rich galaxies, as well as the UCHVC dwarfs in the current work (represented by color bands). The SHIELD (Cannon et al. 2011; McQuinn et al. 2015) and 'Almost Dark' (Cannon et al.|2015; (Janowiecki et al.|2015) samples of gas rich galaxies are described in Section 4.4 Note the very high $M_{H I} / M_{\text {star }}$ for ALFALFA-Dw1. 
TABLE 1

Summary of UCHVC Archival SEARCh

\begin{tabular}{|c|c|c|c|c|c|c|}
\hline $\begin{array}{c}\text { Survey } \\
\text { ID }\end{array}$ & $\begin{array}{c}\text { RA } \\
(\mathrm{J} 2000)\end{array}$ & $\begin{array}{c}\text { DEC } \\
(\mathrm{J} 2000)\end{array}$ & $\begin{array}{l}\mathrm{HI} v_{L S R} \\
\left(\mathrm{~km} \mathrm{~s}^{-1}\right)\end{array}$ & $\begin{array}{c}\text { Optical } \\
\text { Archives }^{\mathrm{a}}\end{array}$ & $\begin{array}{l}\text { GALEX } \\
\text { Archive }\end{array}$ & Comments \\
\hline \multicolumn{7}{|c|}{ GALFA-HI Survey } \\
\hline $003.7+10.8+236$ & $00: 14: 45$ & $+10: 49: 00$ & 235.38 & SDSS & $\mathrm{NUV}^{\mathrm{b}}$ & GALFA-Dw1* \\
\hline $019.8+11.1+617$ & $01: 19: 13$ & $+11: 07: 00$ & 611.63 & SDSS & NUV, FUV ${ }^{\mathrm{b}}$ & GALFA-Dw $2^{*}$ \\
\hline $044.7+13.6+528$ & $02: 58: 57$ & $+13: 37: 00$ & 528.59 & $\operatorname{DSS}^{\mathrm{d}}$ & NUV, FUV ${ }^{b}$ & GALFA-Dw3* \\
\hline $063.7+33.3+447$ & $04: 14: 57$ & $+33: 18: 00$ & 447.77 & DSS & NUV, FUV ${ }^{\mathrm{b}}$ & \\
\hline $084.4+24.0+152$ & $05: 37: 33$ & $+24: 02: 00$ & 153.04 & DSS & $\mathrm{NUV}^{\mathrm{b}}$ & \\
\hline $086.4+31.8+612$ & $05: 45: 45$ & $+31: 46: 00$ & 612.27 & DSS & $\ldots$ & \\
\hline $086.4+10.8+611$ & $05: 45: 45$ & $+10: 46: 00$ & 614.53 & $\operatorname{DSS}^{\mathrm{d}}$ & $\ldots$ & GALFA-Dw $4^{*}$ \\
\hline $090.9+30.9+266$ & $06: 03: 37$ & $+30: 53: 00$ & 267.56 & DSS & $\mathrm{NUV}^{\mathrm{b}}$ & \\
\hline $092.1+09.5+584$ & $06: 08: 33$ & $+09: 29: 00$ & 584.58 & $\operatorname{CFHT}(r)^{e}$ & $\ldots$ & \\
\hline $100.0+36.7+417$ & $06: 39: 49$ & $+36: 41: 00$ & 416.43 & SDSS & NUV,FUV ${ }^{\mathrm{b}}$ & \\
\hline $100.9+09.2+311$ & $06: 43: 33$ & $+09: 13: 00$ & 310.11 & SDSS & $\ldots$ & \\
\hline $104.4+04.1-145$ & $06: 57: 37$ & $+04: 07: 00$ & -157.20 & DSS & $\ldots$ & \\
\hline $143.7+12.9+223$ & 09:34:41 & $+12: 51: 00$ & 222.93 & SDSS & $\mathrm{NUV}^{\mathrm{b}}$ & \\
\hline $147.0+07.1+525$ & 09:48:05 & $+07: 08: 00$ & 526.31 & SDSS & NUV, FUV ${ }^{\mathrm{c}}$ & see Appendix \\
\hline $162.1+12.5+434$ & $10: 48: 25$ & $+12: 31: 00$ & 435.67 & $\operatorname{SDSS}, \mathrm{CFHT}(\mathrm{i})^{\mathrm{g}}$ & NUV, FUV & counterpart with $\Delta \mathrm{v} \sim 5260 \mathrm{~km} \mathrm{~s}^{-1}$ \\
\hline $183.0+04.4-112$ & $12: 12: 10$ & $+04: 23: 00$ & -112.84 & SDSS & $\mathrm{NUV}^{\mathrm{v}}$ & \\
\hline $184.8+05.7-092$ & $12: 19: 06$ & $+05: 40: 00$ & -89.90 & $\operatorname{SDSS}, \operatorname{CFHT}(\mathrm{u}, \mathrm{g}, \mathrm{i})^{\mathrm{h}}$ & NUV, FUV ${ }^{\mathrm{w}}$ & \\
\hline $187.5+08.0+473$ & $12: 29: 54$ & $+07: 58: 00$ & 473.70 & $\operatorname{SDSS}, \operatorname{CFHT}(u, g, i, z)^{\mathrm{i}}$ & NUV, FUV ${ }^{\mathrm{b}}$ & see Appendix \\
\hline $188.9+14.5+387$ & $12: 35: 26$ & $+14: 30: 00$ & 403.41 & SDSS & NUV, FUV ${ }^{\mathrm{b}}$ & see Appendix \\
\hline $195.9+06.9-100$ & $13: 03: 38$ & $+06: 55: 00$ & -104.44 & SDSS & $\mathrm{NUV}, \mathrm{FUV}^{\mathrm{b}}$ & \\
\hline $196.6+06.5-105$ & $13: 06: 18$ & $+06: 29: 00$ & -103.55 & SDSS & NUV $^{\mathrm{x}}$ & \\
\hline $215.9+04.6+205$ & $14: 23: 26$ & $+04: 33: 00$ & 217.30 & SDSS & NUV, FUV ${ }^{\mathrm{c}}$ & HVC351.17+58.56+214 \\
\hline $331.8+21.0+303$ & $22: 07: 06$ & $+20: 59: 00$ & 303.48 & $\operatorname{SDSS}, \operatorname{CFHT}(r)^{\mathrm{j}}$ & $\ldots$ & \\
\hline $339.0+09.0-237$ & $22: 36: 06$ & $+09: 02: 00$ & -236.64 & SDSS & $\mathrm{NUV}^{\mathrm{c}}$ & \\
\hline $341.7+07.7-234$ & $22: 46: 58$ & $+07: 41: 00$ & -234.09 & SDSS & $\ldots$ & \\
\hline $342.1+20.6+208$ & $22: 48: 26$ & $+20: 33: 00$ & 208.14 & SDSS & $\mathrm{NUV}^{\mathrm{c}}, \mathrm{FUV}^{\mathrm{b}}$ & \\
\hline $345.0+07.0-245$ & $22: 59: 58$ & $+07: 01: 00$ & -244.33 & SDSS & NUV, FUV ${ }^{\mathrm{b}}$ & \\
\hline \multicolumn{7}{|c|}{ ALFALFA Survey } \\
\hline $111.65-30.53-124$ & $00: 05: 54.3$ & $+31: 20: 14$ & -124 & SDSS & $\mathrm{NUV}^{\mathrm{b}}$ & \\
\hline $123.11-33.67-176$ & $00: 52: 06.2$ & $+29: 12: 04$ & -176 & SDSS & NUV,FUV ${ }^{\mathrm{b}}$ & \\
\hline $123.74-33.47-289$ & $00: 54: 31.6$ & $+29: 24: 02$ & -289 & SDSS & NUV,FUV ${ }^{\mathrm{b}}$ & \\
\hline $126.85-46.66-310$ & $01: 02: 37.8$ & $+16: 07: 52$ & -310 & SDSS & NUV,FUV ${ }^{\mathrm{b}}$ & \\
\hline $131.90-46.50-276$ & $\begin{array}{llll}01 & 17 & 03.4\end{array}$ & $+15: 55: 48$ & -276 & $\operatorname{SDSS}^{\mathrm{d}}$ & NUV,FUV ${ }^{\mathrm{b}}$ & \\
\hline $137.90-31.73-327$ & $01: 49: 52.1$ & $+29: 26: 00$ & -327 & $\operatorname{SDSS}, \mathrm{CFHT}(\mathrm{r})^{\mathrm{k}}$ & NUV,FUV ${ }^{\mathrm{b}}$ & \\
\hline $138.39-32.71-320$ & $01: 50: 31.4$ & $+28: 22: 59$ & -320 & $\operatorname{SDSS}, \operatorname{CFHT}(\mathrm{r})^{1}$ & NUV,FUV ${ }^{\mathrm{b}}$ & \\
\hline $154.00-29.03-141$ & $02: 52: 29.7$ & $+26: 26: 30$ & -141 & DSS & $\ldots$ & \\
\hline $196.50+24.42+146$ & $07: 55: 27.1$ & $+24: 41: 43$ & 146 & SDSS & NUV,FUV ${ }^{b}$ & \\
\hline $196.09+24.74+166$ & $07: 56: 14.8$ & $+25: 09: 00$ & 166 & SDSS & NUV,FUV ${ }^{\mathrm{c}}$ & B14 \\
\hline $198.48+31.09+165$ & $08: 25: 46.7$ & $+25: 11: 28$ & 165 & SDSS & NUV,FUV ${ }^{\mathrm{b}}$ & \\
\hline $204.88+44.86+147$ & $09: 30: 13.2$ & $+24: 12: 17$ & 147 & SDSS & $\mathrm{NUV}, \mathrm{FUV}^{\mathrm{b}}$ & B14 \\
\hline $205.28+18.70+150$ & $07: 45: 59.9$ & $+14: 58: 37$ & 150 & $\operatorname{SDSS}, \operatorname{CFHT}(\mathrm{r})^{\mathrm{m}}$ & NUV,FUV ${ }^{\mathrm{b}}$ & B14 \\
\hline $234.33+51.28+143$ & $10: 27: 01.1$ & $+08: 47: 08$ & 143 & $\operatorname{SDSS}, \operatorname{CFHT}(\mathrm{g})^{\mathrm{n}}$ & $\ldots$ & \\
\hline $252.98+60.17+142$ & $11: 21: 19.6$ & $+06: 21: 32$ & 142 & SDSS & $\cdots$ & \\
\hline $245.26+69.53+217$ & 11:40:08.1 & $+15: 06: 44$ & 217 & SDSS & $\ldots$ & B14 \\
\hline $255.76+61.49+181$ & $11: 28: 55.6$ & $+06: 25: 29$ & 181 & SDSS & $\mathrm{NUV}, \mathrm{FUV}^{\mathrm{b}}$ & B14 \\
\hline $274.68+74.70-123$ & $12: 21: 54.7$ & $+13: 28: 10$ & -123 & $\operatorname{SDSS}^{\mathrm{d}}, \mathrm{CFHT}(\mathrm{u}, \mathrm{g}, \mathrm{i}, \mathrm{z})^{\mathrm{i}}$ & $\mathrm{NUV}^{\mathrm{y}}$ & ALFALFA-Dw1; B14 \\
\hline $290.19+70.86+204$ & $12: 34: 40.2$ & $+08: 24: 08$ & 204 & $\operatorname{SDSS}, \mathrm{CFHT}(\mathrm{u}, \mathrm{g}, \mathrm{r}, \mathrm{i}, \mathrm{z})^{\mathrm{o}}$ & NUV,FUV ${ }^{b}$ & $\mathrm{~B} 14$ \\
\hline $292.94+70.42+159$ & $12: 37: 58.5$ & $+07: 48: 49$ & 159 & $\operatorname{SDSS}, \operatorname{CFHT}(\mathrm{g}, \mathrm{r}, \mathrm{i}, \mathrm{z})^{\mathrm{p}}$ & $\mathrm{NUV}^{\mathrm{z}}$ & \\
\hline $028.09+71.86-144$ & $14: 10: 58.1$ & $+24: 12: 04$ & -144 & SDSS & NUV,FUV ${ }^{\mathrm{b}}$ & B14 \\
\hline $253.04+61.98+148$ & $11: 26: 24.8$ & $+07: 39: 15$ & 148 & SDSS & NUV,FUV & \\
\hline $256.34+61.37+166$ & $11: 29: 28.6$ & $+06: 09: 23$ & 166 & SDSS & NUV,FUV ${ }^{\mathrm{c}}$ & \\
\hline $250.16+57.45+139$ & $11: 09: 29.8$ & $+05: 26: 01$ & 139 & SDSS & NUV,FUV ${ }^{\mathrm{c}}$ & B14 \\
\hline $277.25+65.14-140$ & $12: 09: 20.0$ & $+04: 23: 30$ & -140 & SDSS & $\mathrm{NUV}^{\mathrm{c}}$ & B14 \\
\hline $295.19+72.63+225$ & $12: 42: 04.6$ & $+09: 54: 05$ & 225 & $\operatorname{SDSS}, \operatorname{CFHT}(\mathrm{g}, \mathrm{r}, \mathrm{i}, \mathrm{z})^{\mathrm{q}}$ & NUV ${ }^{\text {aa }}$ & \\
\hline $298.95+68.17+270$ & $12: 45: 29.8$ & $+05: 20: 23$ & 270 & SDSS & $\mathrm{NUV}^{\mathrm{c}}$ & B14 \\
\hline $320.95+72.32+185$ & $13: 13: 21.5$ & $+10: 12: 57$ & 185 & SDSS & $\mathrm{NUV}^{\mathrm{b}}$ & see Appendix \\
\hline $324.03+75.51+135$ & $13: 12: 42.3$ & $+13: 30: 46$ & 135 & SDSS & NUV,FUV ${ }^{\mathrm{b}}$ & B14 \\
\hline $326.91+65.25+316$ & $13: 30: 43.8$ & $+04: 13: 38$ & 316 & SDSS & $\mathrm{NUV}^{\mathrm{c}}$ & B14 \\
\hline $330.13+73.07+132$ & $13: 22: 41.6$ & $+11: 52: 31$ & 132 & SDSS & NUV,FUV ${ }^{\mathrm{b}}$ & $\mathrm{B} 14$ \\
\hline $351.17+58.56+214$ & $14: 23: 21.2$ & $+04: 34: 37$ & 214 & $\operatorname{SDSS}^{\mathrm{d}}$ & NUV,FUV ${ }^{\mathrm{b}}$ & GALFA $215.9+04.6+205 ;$ B 14 \\
\hline $352.45+59.06+263$ & $14: 23: 57.7$ & $+05: 23: 40$ & 263 & SDSS & $\ldots$ & B14 \\
\hline $353.41+61.07+257$ & $14: 19: 48.6$ & $+07: 11: 15$ & 257 & SDSS & NUV,FUV ${ }^{\mathrm{b}}$ & $\mathrm{B} 14$ \\
\hline $356.81+58.51+148$ & $14: 31: 58.8$ & $+06: 35: 20$ & 148 & SDSS & NUV,FUV ${ }^{b}$ & $\mathrm{~B} 14$ \\
\hline $005.58+52.07+163$ & $15: 04: 41.3$ & $+06: 12: 59$ & 163 & SDSS & NUV,FUV & B14 \\
\hline $013.59+54.52+169$ & $15: 07: 23.0$ & $+11: 32: 56$ & 169 & SDSS & $\mathrm{NUV}, \mathrm{FUV}^{\mathrm{b}}$ & B14 \\
\hline $013.60+54.23+179$ & $15: 08: 24.4$ & $+11: 24: 22$ & 179 & SDSS & NUV,FUV ${ }^{\mathrm{bc}}$ & $\mathrm{B} 14$ \\
\hline $013.63+53.78+222$ & $15: 10: 00.6$ & $+11: 11: 27$ & 222 & SDSS & NUV,FUV ${ }^{\mathrm{c}}$ & B14 \\
\hline $026.01+45.52+161$ & $15: 55: 07.5$ & $+14: 29: 29$ & 161 & SDSS & NUV,FUV ${ }^{\mathrm{ab}}$ & $\mathrm{B} 14$ \\
\hline $026.11+45.88+163$ & $15: 53: 54.0$ & $+14: 41: 48$ & 163 & SDSS & NUV,FUV ${ }^{\mathrm{ab}}$ & \\
\hline $028.07+43.42+150$ & $16: 05: 32.6$ & $+14: 59: 20$ & 150 & SDSS & NUV,FUV ${ }^{\mathrm{ac}}$ & B14 \\
\hline $028.47+43.13+177$ & $16: 07: 07.0$ & $+15: 08: 31$ & 177 & SDSS & NUV,FUV ${ }^{\mathrm{b}}$ & \\
\hline $029.55+43.88+175$ & $16: 05: 29.4$ & $+16: 09: 12$ & 175 & SDSS & NUV,FUV ${ }^{\mathrm{b}}$ & \\
\hline
\end{tabular}


TABLE 1

Summary of UCHVC Archival Search

$\begin{array}{lllccc}028.03+41.54+127 & 16: 12: 36.8 & +14: 12: 27 & 127 & \text { SDSS } & \text { NUV,FUV }^{\mathrm{b}} \\ 028.66+40.38+125 & 16: 17: 45.3 & +14: 10: 36 & 125 & \text { SDSS } & \text { NUV,FUV }^{\mathrm{b}} \\ 019.13+35.24-123 & 16: 22: 35.7 & +05: 08: 48 & -123 & \text { SDSS } & \text { NUV,FUV }^{\mathrm{b}} \\ 027.86+38.25+124 & 16: 24: 43.4 & +12: 44: 12 & 207 & \text { SDSS } & \text { NUV,FUV }^{\mathrm{c}} \\ 080.69-23.84-334 & 22: 01: 00.7 & +24: 44: 04 & -334 & \text { SDSS } & \text { NUV,FUV }^{\mathrm{b}} \\ 082.91-20.46-426 & 21: 58: 02.9 & +28: 37: 35 & -426 & \text { SDSS } & \text { NUV,FUV }^{\mathrm{b}} \\ 082.91-25.55-291 & 22: 12: 38.6 & +24: 43: 11 & -291 & \text { SDSS,CFHT }(\mathrm{r})^{\mathrm{r}} & \text { NUV,FUV }^{\mathrm{b}} \\ 084.01-17.95-311 & 21: 54: 06.2 & +31: 12: 49 & -311 & \text { DSS } & \text { NUV }^{\mathrm{c}} \mathrm{FUV}^{\mathrm{b}} \\ 084.61-26.89-330 & 22: 21: 34.4 & +24: 36: 38 & -330 & \text { SDSS } & \text { NUV,FUV }^{\mathrm{b}} \\ 086.18-21.32-277 & 22: 11: 21.8 & +29: 54: 02 & -277 & \text { SDSS,CFHT }(\mathrm{r})^{\mathrm{s}} & \text { NUV,FUV }^{\mathrm{b}} \\ 087.35-39.78-434 & 23: 00: 56.4 & +15: 20: 14 & -454 & \text { SDSS } & \cdots \\ 088.15-39.37-445 & 23: 02: 11.3 & +16: 00: 48 & -445 & \text { SDSS,CFHT }(\mathrm{u}, \mathrm{g}, \mathrm{r}, \mathrm{i})^{\mathrm{t}} & \ldots \\ 092.53-23.02-311 & 22: 38: 23.4 & +31: 52: 57 & -311 & \text { SDSS } & \text { NUV,FUV }^{\mathrm{b}} \\ 108.98-31.85-328 & 23: 56: 58.8 & +29: 32: 35 & -328 & \text { SDSS } & \text { NUV }^{\mathrm{b}} \\ 109.07-31.59-324 & 23: 57: 02.1 & +29: 48: 46 & -324 & \text { SDSS } & \text { NUV }^{\mathrm{b}}\end{array}$

Note. - Fields with 'B14' noted in the comments are fields with deep imaging reported by Bellazzini et al. (2014). None of these found dwarf counterparts, besides ALFALFA-Dw1

${ }^{*}$ Candidate diffuse dwarf galaxy at UCHVC position. GALFA-Dw1 and GALFA-Dw2 are Pisces A and Pisces B, as reported by T15. ALFALFADw1 is SECCO 1 as reported by Bellazzini et al. (2015). ${ }^{a}$ DSS images are listed only when it is the only option. ${ }^{b}$ From the GALEX All Sky Imaging Survey; $t_{\text {exp }} \sim 100 \mathrm{~s}$ of seconds. ${ }^{c}$ From the GALEX Medium Imaging Survey; $t_{\text {exp }} \sim 1500 \mathrm{~s} .{ }^{d}$ Supplementary imaging taken; see Section 2.2 and Table $2{ }^{e}$ CFHT; r $t_{\exp }=150 \mathrm{~s} .{ }^{f}$ GALEX Guest Investigator data; NUV,FUV $t_{\exp }=1667 \mathrm{~s} .{ }^{g} \mathrm{CFHT}$ i-band $t_{\exp }=476 \mathrm{~s} .{ }^{h} \mathrm{CFHT}(\mathrm{u}, \mathrm{g}, \mathrm{i})$ $t_{\text {exp }}=(2400,2760,3240) \mathrm{s} .{ }^{i} \mathrm{CFHT}(\mathrm{u}, \mathrm{g}, \mathrm{i}, \mathrm{z}) t_{\text {exp }}=(6402,3170,2055,4400) \mathrm{s} .{ }^{j} \mathrm{CFHT} \mathrm{r} t_{\text {exp }}=420 \mathrm{~s} .{ }^{k} \mathrm{CFHT} \mathrm{r} t_{\text {exp }}=1600 \mathrm{~s} .{ }^{l} \mathrm{CFHT} \mathrm{r}$ $t_{e x p}=2260 \mathrm{s.} .{ }^{m} \mathrm{CFHT} \mathrm{r} t_{e x p}=200 \mathrm{~s} .{ }^{n} \mathrm{CFHT} \mathrm{g} t_{e x p}=1050 \mathrm{~s} .{ }^{\circ} \mathrm{CFHT}(\mathrm{u}, \mathrm{g}, \mathrm{r}, \mathrm{i}, \mathrm{z}) t_{\text {exp }}=(6800,3100,3720,6000,7500) \mathrm{s} .{ }^{p} \mathrm{CFHT}(\mathrm{g}, \mathrm{r}, \mathrm{i}, \mathrm{z})$ $t_{\text {exp }}=(3170,4461,2055,4400) \mathrm{s} .{ }^{q} \mathrm{CFHT}(\mathrm{g}, \mathrm{r}, \mathrm{i}, \mathrm{z}) t_{\text {exp }}=(4800,1400,7500,4400) \mathrm{s} .{ }^{s} \mathrm{CFHT} \mathrm{r} t_{\text {exp }}=480 \mathrm{~s} .{ }^{t} \mathrm{CFHT} \mathrm{r} t_{\text {exp }}=240 \mathrm{~s},{ }^{u} \mathrm{CFHT}(\mathrm{u}, \mathrm{g}, \mathrm{r}, \mathrm{i})$ $t_{\exp }=(2800,5400,920,470) \mathrm{s},{ }^{v}$ GALEX Guest Investigator data; NUV $t_{e x p}=1730 \mathrm{~s} .{ }^{w}$ GALEX Guest Investigator data; FUV,NUV $t_{e x p}=1655$, $1655 \mathrm{~s},{ }^{x}$ GALEX Guest Investigator data; NUV $t_{e x p}=1650 \mathrm{~s} .,{ }^{y}$ GALEX Guest Investigator data; NUV $t_{e x p}=1690 \mathrm{~s} .{ }^{z}$ GALEX Guest Investigator data; NUV $t_{\exp }=1605 \mathrm{s.}^{a a}$ GALEX Guest Investigator data; NUV $t_{e x p}=1909 \mathrm{s.}{ }^{a b}$ GALEX Guest Investigator data; FUV, NUV $t_{e x p}=1500,1500$ s. ${ }^{a c}$ GALEX Guest Investigator data; FUV, NUV $t_{\text {exp }}=5057,5057 \mathrm{~s}$. 
TABLE 2

Supplementary Imaging LoG

\begin{tabular}{|c|c|c|c|c|c|c|}
\hline Target & $\begin{array}{l}\text { Telescope/ } \\
\text { Instrument }\end{array}$ & UT Date & Filter & $\begin{array}{l}\text { Exposure } \\
\quad(\mathrm{sec})\end{array}$ & $\begin{array}{l}\text { Depth } \\
\text { (mag) }\end{array}$ & Notes \\
\hline HVC2 $24.68+74.70-123$ & Magellan/Megacam & 11 June 2013 & $\begin{array}{l}\mathrm{g} \\
\mathrm{r}\end{array}$ & $\begin{array}{l}8 \times 300 \\
6 \times 300\end{array}$ & $\begin{array}{l}25.0 \\
25.1\end{array}$ & ALFALFA-Dw1 \\
\hline HVC351.17+58.56+214 & Magellan/Megacam & 27 April 2014 & $\begin{array}{l}\mathrm{g} \\
\mathrm{r}\end{array}$ & $\begin{array}{l}6 \times 300 \\
6 \times 300\end{array}$ & $\begin{array}{l}25.9 \\
25.9\end{array}$ & \\
\hline GALFA $044.7+13.6+528$ & Magellan/Megacam & 26 Oct 2014 & $\begin{array}{l}\mathrm{g} \\
\mathrm{r}\end{array}$ & $\begin{array}{l}7 \times 300 \\
8 \times 300\end{array}$ & $\begin{array}{l}25.3 \\
25.5\end{array}$ & GALFA-Dw3 \\
\hline HVC131.90-46.50-276 & APO/SPICAM & 17 Nov 2014 & $\begin{array}{l}\mathrm{g} \\
\mathrm{r}\end{array}$ & $\begin{array}{l}2 \times 540 \\
2 \times 540\end{array}$ & $\begin{array}{l}23.3 \\
23.5\end{array}$ & \\
\hline GALFA $086.4+10.8+611$ & APO/SPICAM & 17 Nov 2014 & $\begin{array}{l}\mathrm{g} \\
\mathrm{r}\end{array}$ & $\begin{array}{l}4 \times 450 \\
4 \times 450\end{array}$ & $\begin{array}{l}23.6 \\
23.8\end{array}$ & GALFA-Dw4 \\
\hline
\end{tabular}

b Also identified as GALFA 215.9+04.6+205 
TABLE 3

SPECTROSCOPY LOG

\begin{tabular}{llcccc}
\hline \hline \multicolumn{1}{c}{ Target } & \multicolumn{1}{c}{$\begin{array}{c}\text { UCHVC } \\
\text { ID }\end{array}$} & $\begin{array}{c}\text { Telescope/ } \\
\text { Instrument }\end{array}$ & UT Date & $\begin{array}{c}\text { Exposure } \\
(\mathrm{sec})\end{array}$ & $\begin{array}{c}\text { H } \alpha v_{L S R} \\
\left(\mathrm{~km} \mathrm{~s}^{-1}\right)\end{array}$ \\
\hline ALFALFA-Dw1 & HVC274.68+74.70-123 & Magellan/IMACS & 17 June 2014 & $5 \times 1800$ & $-114 \pm 12$ \\
GALFA-Dw1 & GALFA 003.7+10.8+236 & SOAR/Goodman & 29 June 2014 & $5 \times 900$ & $236 \pm 8$ \\
GALFA-Dw2 & GALFA 019.8+11.1+617 & MMT/BCS & 21 Oct 2014 & $1 \times 900$ & $625 \pm 30$ \\
GALFA-Dw3 & GALFA 044.7+13.6+528 & APO/DIS & 18 Nov 2014 & $5 \times 1200$ & $503 \pm 35$ \\
GALFA-Dw4 & GALFA 086.4+10.8+611 & APO/DIS & 18 Nov 2014 & $3 \times 1200$ & $607 \pm 35$ \\
SDSS J1048+1230 & GALFA 162.1+12.5+434 & MMT/BCS & 24 Dec 2014 & $2 \times 900$ & $5730 \pm 200$ \\
& & & & Not ass. with UCHVC \\
\hline
\end{tabular}


TABLE 4

Properties of the New Dwarf Galaxies

\begin{tabular}{|c|c|c|c|c|c|}
\hline Dwarf & GALFA-Dw $1^{a}$ & GALFA-Dw $2^{b}$ & GALFA-Dw3 & GALFA-Dw4 & ALFALFA-Dw $1^{c}$ \\
\hline Survey ID & $003.7+10.8+236$ & $019.8+11.1+617$ & $044.7+13.6+528$ & $086.4+10.8+611$ & $274.68+74.70-123$ \\
\hline RA (J2000) (optical) & $00: 14: 46.09$ & 01:19:11.72 & $02: 58: 56.24$ & $05: 45: 44.79$ & $12: 21: 54.02$ \\
\hline DEC (J2000) (optical) & $+10: 48: 47.1$ & $+11: 07: 16.3$ & $+13: 37: 47.9$ & $+10: 46: 15.6$ & $+13: 27: 37.32$ \\
\hline $1\left({ }^{\circ}\right)$ & 108.52 & 133.83 & 164.14 & 195.66 & 274.68 \\
\hline $\mathrm{b}\left({ }^{\circ}\right)$ & -51.03 & -51.16 & -38.83 & -9.32 & 74.69 \\
\hline $\mathrm{E}(\mathrm{B}-\mathrm{V})(\mathrm{mag})$ & 0.09 & 0.05 & 0.13 & 0.54 & 0.05 \\
\hline $\mathrm{HI} v_{L S R}\left(\mathrm{~km} \mathrm{~s}^{-1}\right)$ & 235.38 & 611.63 & 528.59 & 614.53 & -123 \\
\hline$H \alpha v_{L S R}\left(\mathrm{~km} \mathrm{~s}^{-1}\right)$ & $236 \pm 8$ & $625 \pm 30$ & $503 \pm 35$ & $607 \pm 35$ & $-114 \pm 12$ \\
\hline$r_{0}(\mathrm{mag})$ & $17.12 \pm 0.19$ & $16.91 \pm 0.13$ & $16.43 \pm 0.32$ & $14.52 \pm 0.08$ & $20.51 \pm 0.26$ \\
\hline$(g-r)_{0}(\mathrm{mag})$ & $0.19 \pm 0.22$ & $0.09 \pm 0.16$ & $0.32 \pm 0.32$ & $0.06 \pm 0.14$ & $-0.10 \pm 0.35$ \\
\hline$N U V_{0}$ (mag) & $18.48 \pm 0.15$ & $18.50 \pm 0.19$ & $17.58 \pm 0.60$ & $\begin{array}{l}0.00 .11 \\
\ldots\end{array}$ & $20.73 \pm 0.70$ \\
\hline$F U V_{0}$ (mag) & $\ldots$ & $18.64 \pm 0.44$ & $17.57 \pm 0.38$ & $\cdots$ & $\ldots$ \\
\hline$r_{h}(\operatorname{arcsec})$ & $8.6 \pm 1.8$ & $10.2 \pm 2.6$ & $9.2 \pm 1.5$ & $12.5 \pm 0.6$ & $8.1 \pm 0.9$ \\
\hline$F_{H I}\left(\mathrm{Jy} \mathrm{km} \mathrm{s}^{-1}\right)$ & $1.22 \pm 0.07^{\mathrm{d}}$ & $1.6 \pm 0.2^{\mathrm{d}}$ & $1.7 \pm 0.1$ & $1.89 \pm 0.25$ & $0.92^{\mathrm{e}}$ \\
\hline \multicolumn{6}{|c|}{ Distance Dependent Properties } \\
\hline Est. Dist range (Mpc) & $1.7-5.3$ & 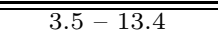 & 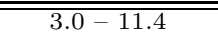 & 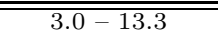 & 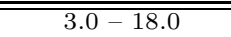 \\
\hline$M_{r}(\mathrm{mag})$ & -9.0 to -11.5 & -10.8 to -13.7 & -10.9 to -13.8 & -12.9 to -16.1 & -6.9 to -10.8 \\
\hline$r_{h}(\mathrm{pc})$ & $71-220$ & $173-663$ & $133-510$ & $181-806$ & $117-707$ \\
\hline$M_{*}\left(10^{5} M_{\odot}\right)$ & $3.3-33.0$ & $18.1-265.6$ & $19.0-276.2$ & $121.7-2392.0$ & $0.5-18.7$ \\
\hline$M_{H I}\left(10^{5} M_{\odot}\right)$ & $8.3-80.6$ & $46.2-677.9$ & $36.0-524.3$ & $40.0-787.8$ & $19.5-702.3$ \\
\hline
\end{tabular}

a Alternatively named Pisces A by T15

b Alternatively named Pisces B by T15

C Alternatively named SECCO 1 by B15

d HI flux as reported in T15

e HI flux as reported in A13; no uncertainty presented 